\title{
3D CAA methodology using synthetic turbulence to assess turbulence-cascade interaction noise emission and reduction from serrated airfoils
}

\author{
M. Buszyk*, C. Polacsek ${ }^{\dagger}$ and T. Le Garrec ${ }^{\dagger}$ \\ DAAA, ONERA, Université Paris-Saclay, 92320 - Châtillon, France \\ R. Barrier ${ }^{\S}$ \\ DAAA, ONERA, Université Paris-Saclay, 92190 - Meudon, France \\ C. Bailly ${ }^{\mathrm{II}}$ \\ LMFA, Ecole Centrale de Lyon, 69134 - Ecully, France
}

\begin{abstract}
The context of this study is the development of innovative stator concepts to reduce noise from rotor/stator interaction in future high by-pass engines. Leading edge serrations are examined using a hybrid CFD/CAA method. A stochastic approach is considered for the turbulence generation based on a Fourier modes decomposition. A methodology to obtain a fully 3D turbulence field, while taking into account periodic and wall boundary conditions is developed and a formulation is finally provided to obtain a perfectly incompressible stochastic field. The influence of the turbulence structure on far-field acoustic spectra is first discussed for the baseline computation case. In a second step, numerically assessed noise reductions from the serrated design are favorably compared with an analytical solution and a semi-empirical law. An overall sound power level reduction around 4 to $6 \mathrm{~dB}$ is obtained at three acoustics certification points. Finally, the aerodynamic performances of the serrated airfoil are evaluated through RANS computations and an improved variant of the initial design is proposed, allowing for acceptable penalties at the aerodynamic design point.
\end{abstract}

\section{Nomenclature}

$\begin{array}{ll}\langle\rangle & =\text { Set average operation } \\ i / 1,2,3 & =\text { Indices/subscripts denoting a direction }(i=1 \text {, mean flow direction) } \\ \alpha & =\text { A random angle in }[0,2 \pi] \\ \beta_{c} & =\text { Entrance flow angle (with respect to } x \text { axis) } \\ c & =\text { Chord of the airfoil } \\ E & =\text { Energy spectrum of turbulence } \\ f_{\text {min }}, f_{\text {max }} & =\text { Respectively minimal and maximal considered frequencies } \\ \Delta f & =\text { Frequency spacing } \\ f_{w} & =\text { Window function } \\ g_{w} & =\text { Correction function } \\ h_{s} & =\text { Amplitude of the serrations } \\ \boldsymbol{k} & =\text { Wavevector } \\ k & =\text { Warm of the wavevector } \boldsymbol{k} \\ \boldsymbol{k}^{\perp} & =\text { Coordinates along three orthogonal directions }(1,2,3) \text { of the wavenumber space } \\ \left(k_{1}, k_{2}, k_{3}\right) & =\text { Coordinates of the wavevector } \boldsymbol{k} \text { in the basis }(\boldsymbol{x}, \boldsymbol{y}, \boldsymbol{z}) \\ \left(k_{x}, k_{y}, k_{z}\right) & \end{array}$

\footnotetext{
* PhD candidate, Aerodynamics Aeroelasticity Aeroacoustics Department, martin.buszyk@ onera.fr.

†Senior research engineer, Aerodynamics Aeroelasticity Aeroacoustics Department, cyril.polacsek@onera.fr.

$\doteqdot$ Research engineer, Aerodynamics Aeroelasticity Aeroacoustics Department, thomas.le_garrec@onera.fr.

${ }^{\S}$ Research engineer, Aerodynamics Aeroelasticity Aeroacoustics Department, raphael.Barrier@onera.fr.

IIProfessor, Fluid Mechanics and Acoustics Laboratory, christophe.bailly@ec-lyon.fr, and Senior AIAA Member.
} 


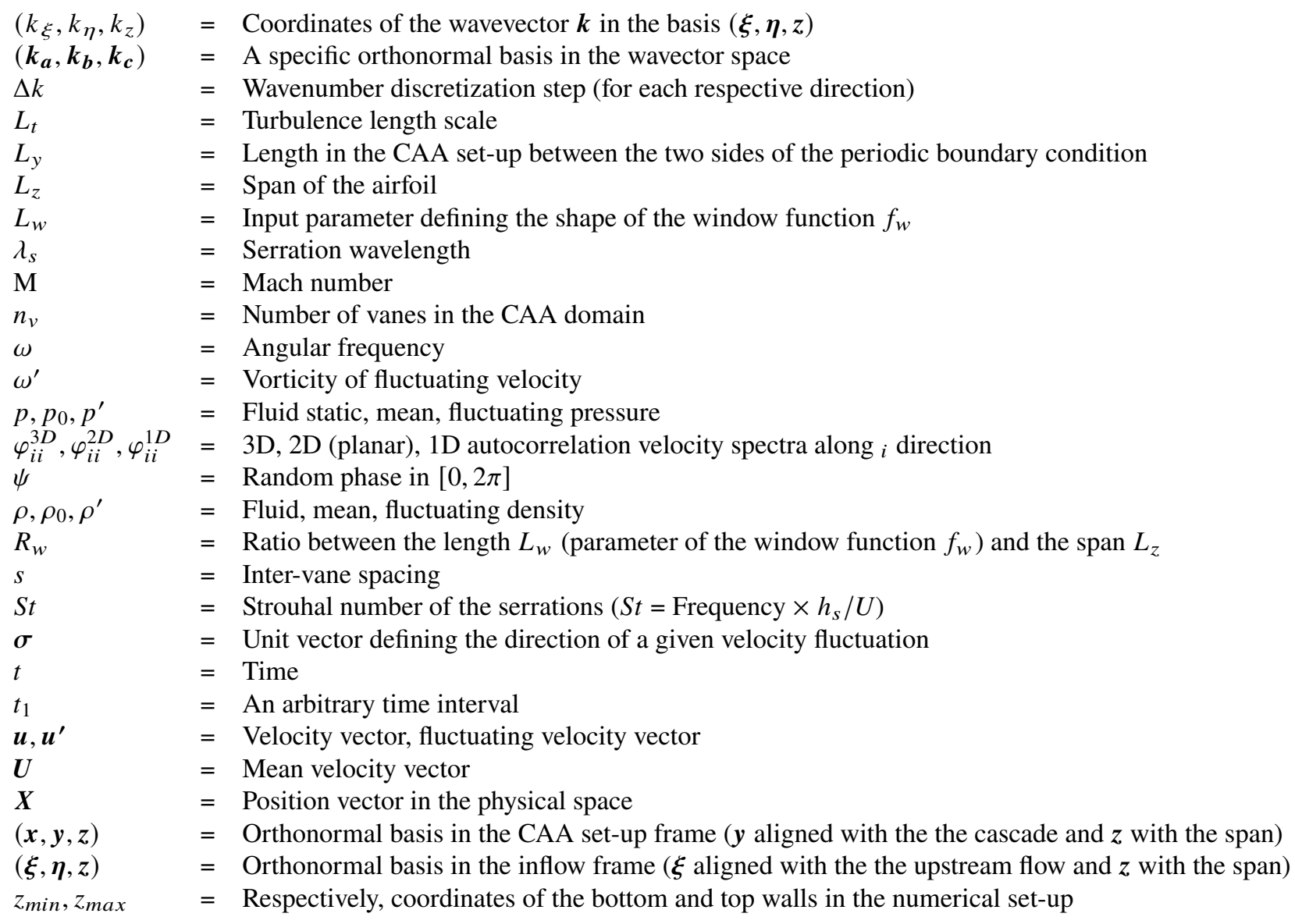

\section{Introduction}

In order to lower the turbofan engine noise emission, much effort is put on damping the rotor/stator interaction noise sources which are the dominant contributions for both tonal and broadband noise components particularly at approach (APP) condition. With this perspective, research projects have been set up such as InnoSTAT in the framework of the H2020 European programs. The goal is to study promising passive and active technologies which might be implemented in future innovative stators. ONERA is involved in the design of a successful passive concept based on leading edge serrations [1, 2]. A set of these serrated low-noise vanes will be evaluated during the first test campaign of the InnoSTAT project which will take place at Ecole Centrale de Lyon (ECL). Two numerical approaches are actually affordable for simulating turbulence-cascade interactions including serrated airfoils. The first one is relying on the Lattice-Boltzmann Method (LBM), as proposed for example in [3], and that is also under study at ONERA [4]. The second one discussed in the present paper, is based on a hybrid CFD/CAA method, involving a synthetic turbulence model. First calculations already performed on the InnoSTAT rectilinear cascade have been reported in [5]. They mainly focused on the determination of the best-suited numerical set-up in particular depending on the number of vanes taken into account for the CAA. Numerical simulations using one-dimensional and planar turbulence structures as inflow condition, have also been realized and first noise reduction evaluations have been performed. The generation of a fully 3D turbulence structure represents still a challenging issue. Several methods have emerged to tackle this issue such as Synthetic Eddy Method (SEM) [6, 7], extended to anisotropic flows and serrated airfoils [8, 9], and Random Particle Mesh (RPM) methodology [10] with recent developments to generate 3D turbulence fields [11, 12]. In this paper, the focus is put on the synthetic turbulence modeling with a particular interest on the development and implementation of a fully three-dimensional vector field (with the complete 3-wavenumbers spectrum and 3-velocity components). The method proposed here to generate synthetic turbulence structures is based on Fourier modes decomposition of the velocity introduced in [13] and [14, 15]. Until now, it has been implemented at ONERA to correctly reproduce the upwash velocity component to the airfoil. That is the dominant component behind turbulence interaction noise mechanism, as shown for example analytically in [16] or through numerical simulations in [8] for serrated airfoils. The 
upwash velocity is represented via its autocorrelation spectrum and through planar turbulent structures. However, as shown in the present work, the latter approach is not directly expandable to 3D turbulence structures. Consequently, the framework used for homogeneous isotopic turbulence (HIT) generation and currently relying on the energy spectrum definition such as proposed in [14] and in [17] is considered. An equivalent mathematical formalism is originally proposed to still link the energy spectrum with wavenumber distribution over a spherical volume to the velocity autocorrelation spectra using a Cartesian writing over a rectangular volume. This equivalence is drawn for both 2D and 3D turbulence structures. This paper follows on previous work at ONERA on CAA with synthetic turbulence based on Fourier modes decomposition [1, 2, 18]. Turbulence generation processes able to tackle some of the issues arising in simulations of practical configurations are implemented, such as wall and periodic boundary conditions (BC), while keeping the CPU time as low as possible. The new methodology is first validated and then applied on both baseline (untreated) and serrated (treated) geometries in the context of the InnoSTAT project. A comparison is performed with previous results and with semi-empirical and analytical solutions. A semi empirical law proposed by Paruchuri et al. [19] is considered to link the power noise reduction to the Strouhal number St of single-wavelength serrations. Moreover, an analytical formulation to assess noise reductions provided by wavy leading edges has also been implemented as detailed in [2]. It was initially developed at University of Cambridge [20], by means of the Wiener-Hopf (WH) technique, and slightly extended and validated by ONERA [2].

The structure of the paper is as follows. Sec. II describes the experimental set-up at ECL. In Sec. III, the aeroacoustic numerical methodology used in this study is detailed. The development and implementation of synthetic turbulence generation routines are summarized in Sec. IV. Issues raised by three-dimensional structures and associated with the boundary conditions of the CAA are highlighted. Sec. V presents CAA computations to assess noise emission on both baseline and serrated geometries. Finally, the aerodynamic performances evaluated by means of RANS computations are discussed in Sec. VI.

\section{Description of the experimental set-up}

The experimental facility (used for the first test campaign of the InnoSTAT project) consists in a rectilinear cascade, depicted in Figs. 1a and $1 \mathrm{~b}$, impinged by a roughly isotropic and homogeneous turbulence flow generated by an adequately shaped turbulence grid placed upstream of the airfoils inside the convergent. Table 1 summarizes the main parameters expected to be representative of the approach condition point at $M=0.34$. Some of the pre-test conditions are being adjusted regarding the definitive cascade set-up specifications. Target values in parenthesizes indicate initial pre-test values used for the present aeroacoustic calculations.

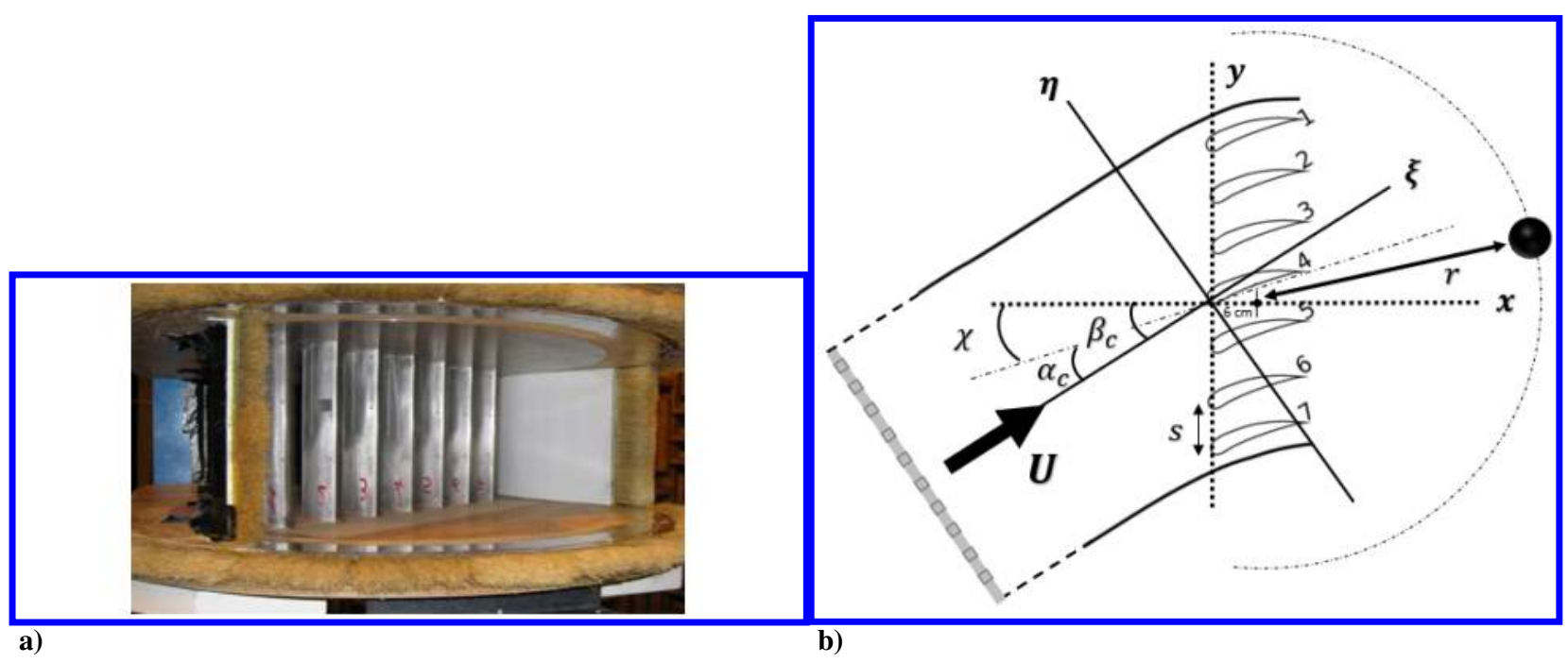

Fig. 1 Test facility for InnoSTAT at Ecole Centrale de Lyon (left) [21] • $(x, y)$ cut of the geometry and coordinate systems (right). 


\begin{tabular}{lll}
\hline \hline & Parameter & Value \\
\hline \multirow{3}{*}{ Airfoil } & shape & NACA7310 \\
& chord $c$ & $12 \mathrm{~cm}$ \\
& span $L_{z}$ & $20 \mathrm{~cm}$ \\
& inter-vane space $s$ & $8.5 \mathrm{~cm}$ \\
\hline \multirow{2}{*}{ Upstream flow } & Mach number $(\boldsymbol{U}$ direction $)$ & $0.34(0.3)$ \\
& Entrance angle $\beta_{c}$ & $34^{\circ}\left(30^{\circ}\right)$ \\
\hline \multirow{2}{*}{ Turbulence } & Turbulence Intensity $(\mathrm{TI})$ & $4.5 \%(5 \%)$ \\
& Turbulent Length Scale $\left(L_{t}\right)$ & $9 \mathrm{~mm}(8 \mathrm{~mm})$ \\
\hline
\end{tabular}

Table 1 Main parameters of the rectilinear cascade configuration at approach condition, in parenthesis, initial pre-test values.

\section{Quick overview of the hybrid computational methodology}

A hybrid method has been implemented at ONERA to conduct aeroacoustic studies, a diagram block is provided in Fig. 2. The main step is the CAA simulation, achieved using an in-house code sAbrinA solving the linearized Euler equations (LEE) for perturbated variables, which are detailed in [22, 23]. The unsteady flow field is classically split in two parts, namely the mean flow $\left(\boldsymbol{U}, p_{0}, \rho_{0}\right)$ which has to be provided as an input, and the fluctuating part $\left(\boldsymbol{u}^{\prime}, p^{\prime}, \rho^{\prime}\right)$ which solution is computed by the code. Regarding numerical schemes, sAbrinA uses a 6th order finite difference scheme for the spatial derivatives and a $3^{\text {rd }}$ order compact explicit Runge-Kutta scheme for the temporal discretization. Specific treatments and boundary conditions (BC) are implemented, such as a 10th order filter in order to remove high-frequency oscillations and Tam boundary conditions [24], which are used both to allow the exit and entrance of the fluctuations in the domain without generating spurious noise sources nor numerical reflections. The mean flow which advects the fluctuating variables is an input parameter of the CAA computations. A RANS computation is usually performed to this end. However, in order to comply with the non viscous assumption of the CAA code, boundary layers have to be removed. To avoid this correction step, the mean flow has been obtained here by means of an open source CFD code solving the Euler equations [25]. Turbulence inflow generated through a stochastic process is injected at the entrance of the CAA domain using Tam's non-reflective boundary condition. The procedure developed to obtain the synthetic turbulence is detailed in the next section. The third step is devoted to computation of sound radiation from an integral formulation. The fluctuating pressure $p_{w a l l}^{\prime}$ is extracted at the vane skin throughout simulation time and radiated in the far-field using a Ffwocs-Williams and Hawkings (FWH) analogy with a Green function valid for a free-space medium with a uniform mean flow. The latter integral method is completed using an in-house code MIA. 


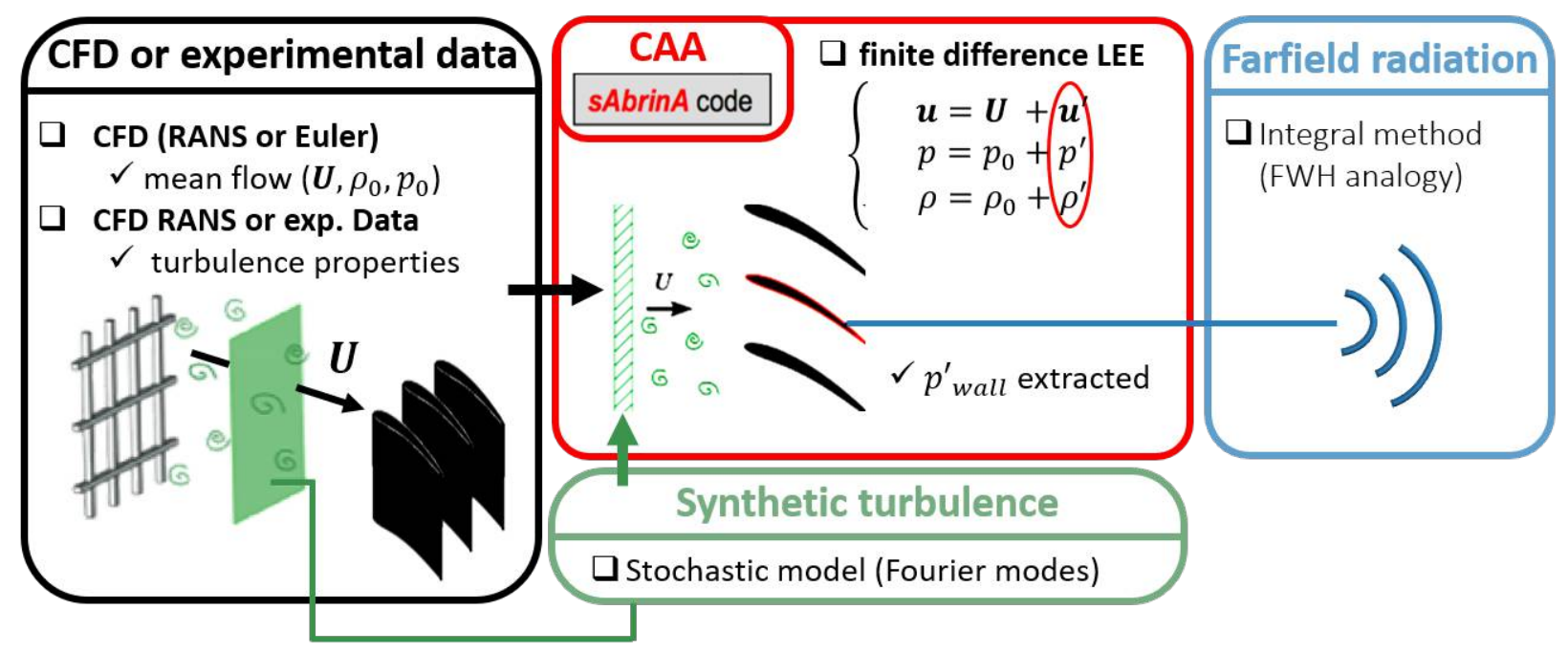

Fig. 2 Main components of the CFD/CAA computation chain.

\section{Synthetic turbulence modeling and generation}

\section{A. Prescribed geometry and main assumptions}

The starting idea to synthesize turbulence is based on an ergodic stochastic process. First, a set average \langle\rangle can be applied on the velocity field in order to get a decomposition in a mean part and a fluctuating part,

$$
\left\{\begin{array}{ccc}
\boldsymbol{u} & = & \boldsymbol{U}+\boldsymbol{u}^{\prime} \\
\boldsymbol{U} & = & \langle\boldsymbol{u}\rangle \\
\left\langle\boldsymbol{u}^{\prime}\right\rangle & = & 0
\end{array}\right.
$$

For the configuration under study here, with almost a uniform mean flow, turbulence can be considered as being statistically steady. This means that the set average operation is equivalent to a time average. Hence, assumed in agreement with previous remark,

$$
\boldsymbol{u}^{\prime}(\boldsymbol{X}, t)=\boldsymbol{u}^{\prime}\left(\boldsymbol{X}+\boldsymbol{U} t_{1}, t+t_{1}\right), \forall t_{1}
$$

The validity of the frozen turbulence hypothesis depends on characteristic times of turbulence and of the advection by the mean flow. They must satisfy $\tau_{\text {turb }} \gg \tau_{\text {conv }}$, that is, $u^{\prime} / U \ll 1$. The frozen turbulence assumption implies that the angular frequency of the velocity fluctuations is directly related to the wavevector thanks to the following dispersion relation $\omega=\boldsymbol{k} \cdot \boldsymbol{U}$. In this study, the wavenumber spectra of turbulence are based on the definition given by Liepmann [26],

$$
E(k)=\frac{8 \mathrm{TI}^{2} U^{2} L_{t}}{\pi} \frac{(k L)^{4}}{\left(1+\left(k L_{t}\right)^{2}\right)^{3}} \text { with } k=\sqrt{k_{1}^{2}+k_{2}^{2}+k_{3}^{2}} .
$$

where the subscript ${ }_{1}$ indicates the direction parallel to the mean flow and TI, the turbulence intensity. From Eq. (2), the link with the autocorrelation velocity spectra can be made through:

$$
\varphi_{i i}^{3 D}\left(k_{1}, k_{2}, k_{3}\right)=\frac{E(k)}{4 \pi k^{2}}\left(1-\frac{k_{i}^{2}}{k^{2}}\right) .
$$

In order to consider simplified cases with planar turbulence and also 1D spectra, an integration is performed over transverse wavenumber components. 


$$
\begin{gathered}
\varphi_{i i}^{2 D}\left(k_{1}, k_{3}\right)=\int_{-\infty}^{+\infty} \varphi_{i i}^{3 D}\left(k_{1}, k_{2}, k_{3}\right) d k_{2} \\
\varphi_{i i}^{1 D}\left(k_{1}\right)=\int_{-\infty}^{+\infty} \varphi_{i i}^{2 D}\left(k_{1}, k_{3}\right) d k_{3}
\end{gathered}
$$

The previous integration in Eqs. (4) and (5) can be analytically performed to obtain explicitly $\varphi_{11}, \varphi_{22}$, and $\varphi_{33}$ which are of major interest for the implementation. They are listed in Table 2.

\begin{tabular}{|l|l|}
\hline 2D planar spectra $\varphi_{i i}^{2 D}\left(k_{1}, k_{3}\right)$ & 1D spectra $\varphi_{i i}^{1 D}\left(k_{1}\right)$ \\
\hline$\varphi_{11}^{2 D}\left(k_{1}, k_{3}\right)=\frac{\mathrm{TI}^{2} U^{2} L_{t}^{2}}{4 \pi} \frac{1+L_{t}^{2}\left(k_{1}^{2}+4 k_{3}^{2}\right)}{\left(1+L_{t}^{2}\left(k_{1}^{2}+k_{3}^{2}\right)\right)^{5 / 2}}$ & $\varphi_{11}^{1 D}\left(k_{1}\right)=\frac{\mathrm{TI}^{2} U^{2} L_{t}}{\pi} \frac{1}{1+\left(L_{t} k_{1}\right)^{2}}$ \\
$\varphi_{22}^{2 D}\left(k_{1}, k_{3}\right)=\frac{3 \mathrm{TI}^{2} U^{2} L_{t}^{4}}{4 \pi} \frac{k_{1}^{2}+k_{3}^{2}}{\left(1+L_{t}^{2}\left(k_{1}^{2}+k_{3}^{2}\right)\right)^{5 / 2}}$ & $\varphi_{22}^{1 D}\left(k_{1}\right)=\frac{\mathrm{TI}^{2} U^{2} L_{t}}{2 \pi} \frac{1+3\left(L_{t} k_{1}\right)^{2}}{\left(1+\left(L_{t} k_{1}\right)^{2}\right)^{2}}$ \\
$\varphi_{33}^{2 D}\left(k_{1}, k_{3}\right)=\frac{\mathrm{TI}^{2} U^{2} L_{t}^{2}}{4 \pi} \frac{1+L_{t}^{2}\left(4 k_{1}^{2}+k_{3}^{2}\right)}{\left(1+L_{t}^{2}\left(k_{1}^{2}+k_{3}^{2}\right)\right)^{5 / 2}}$ & $\varphi_{33}^{1 D}\left(k_{1}\right)=\varphi_{22}^{1 D}\left(k_{1}\right)$
\end{tabular}

Table 2 Integrated autocorrelation spectra obtained from Liepmann spectrum, Eq. (2).

For simplified turbulence structures, one may choose not to consider the influence of the $k_{3}$ component, with the subscript 3 referring to the direction aligned with the span of the studied airfoil or flat plate. Indeed, it has shown to be not significant for flat plates placed along $z$ axis, following Amiet theory under certain hypothesis as detailed in [16, 27]. Spectra are then not explicitly discretized along the latter direction since $k_{3}=k_{z}=0$. To recover the expected magnitude of turbulence, a correction is required by multiplying the spectrum by a factor $\Delta k_{z}=2 \pi / L_{z}$, as proposed in [27]. Beyond these reminders of some assumptions commonly used in turbulence and aeroacoustic modeling, the particular geometrical characteristics and CAA set-up of the rectilinear cascade facility, as depicted in Fig. 3 must be considered. First of all, in order to limit the CPU cost, the test facility is not fully included in the CAA. Adjacent vanes are taken into account through periodic boundary conditions applied along the $y$ direction. Multi-channel calculations can be performed, simply considering that $L_{y}=n_{v} s$. An example is given in Fig. 3 for $n_{v}=1$. The cascade direction that is tilted from the normal axis to the mean flow, as shown by Fig. $1 \mathrm{~b}$, requires to be taken into account in the generation procedure since the gusts are injected in the coordinate system of the cascade $(x, y, z)$ and not the one aligned with the mean flow $(\xi, \eta, z)$. Therefore, to ensure periodicity, all wavenumbers $k_{y}$ have to be multiples of $2 \pi / L_{y}$. Moreover, wall boundary conditions are taken into account for the CAA computations at both ends of the airfoil span. These slip flow boundary conditions considered for the CAA computations represent a major constraint for the generation of a fully $3 \mathrm{D}$ turbulence as explained later.

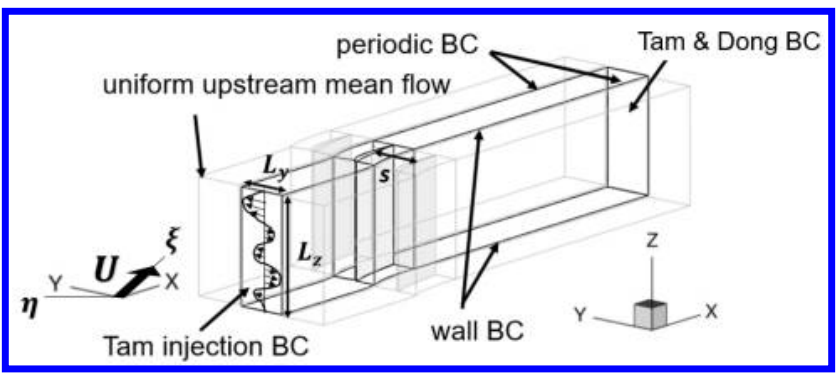

Fig. 3 Boundary conditions for the CAA computation. 


\section{B. Computing planar turbulence structures through velocity autocorrelation spectra}

1. $\left(k_{1}, k_{3}=0\right)$ turbulence structure

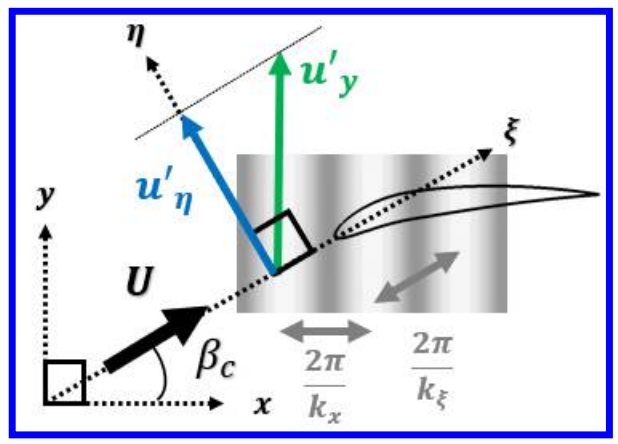

Fig. 4 Coordinate systems with associated wavenumbers and involved turbulent velocities for turbulence structures lying in the plane $\left(k_{1}, k_{3}\right)$.

First of all, let us consider the simplest turbulence field for 3D geometries, namely parallel gust, for which $k_{y}=0$. The divergence free condition leads to the simple condition $\partial u_{x}^{\prime} / \partial x=0$. Thus, $u_{x}^{\prime}$ is constant (here zero) and the overall turbulence velocity is driven by the one-component velocity $u_{y}^{\prime}$. To recover the prescribed normal velocity, $u_{\eta}^{\prime}$, we set $u_{y}^{\prime}=u_{\eta}^{\prime} / \cos \left(\beta_{c}\right)$. The angular frequency is independent of the considered reference frame, therefore $k_{x}=k_{\xi} / \cos \left(\beta_{c}\right)=\omega / U_{x}=\omega /\left(U \cos \left(\beta_{c}\right)\right)$. The dot product is not associated with the choice of the coordinate system, that is why, the spatial phase can be written either $k_{x} x$ or $k_{\xi} \xi$. The spatial discretization step is given by $\Delta k_{\xi, l}=\cos \left(\beta_{c}\right) \Delta k_{x, l}$ which can be associated with the angular frequency discretization through $\omega_{l}=2 \pi l \Delta f$. This equation allows to generate $L$ modes equally distributed from $f_{\min }=\Delta f$ to $f_{\max }=L \Delta f$. For each mode $l$, a random phase $\psi_{l}$ is also introduced. The autocorrelation spectrum of the upwash velocity component is defined by $\varphi_{\eta \eta}^{2 D}\left(k_{\xi}, 0\right)$, refer to $\varphi_{22}^{2 D}\left(k_{1}, k_{3}\right)$ definition in Table 2 Since, the wavenumber $k_{z}$ is not defined, turbulence spectrum has also to be weighted by $\Delta k_{z}=2 \pi / L_{z}$, in order to obtain the prescribed magnitude of the injected turbulence as mentioned in [27].

$$
u_{y}^{\prime}(\boldsymbol{X}, t)=\frac{2}{\cos \left(\beta_{c}\right)} \sum_{l=1}^{L} \sqrt{\varphi_{\eta \eta}^{2 D}\left(k_{\xi, l}, 0\right) \Delta k_{\xi} \frac{2 \pi}{L_{z}}} \cos \left(k_{x, l} x-\omega_{l} t+\psi_{l}\right)
$$

Since the synthetic turbulence (defined by Eq. (6) depends only on the $k_{x}$ wavenumber, the numerical results match exactly the analytical solution as depicted in Fig. 5

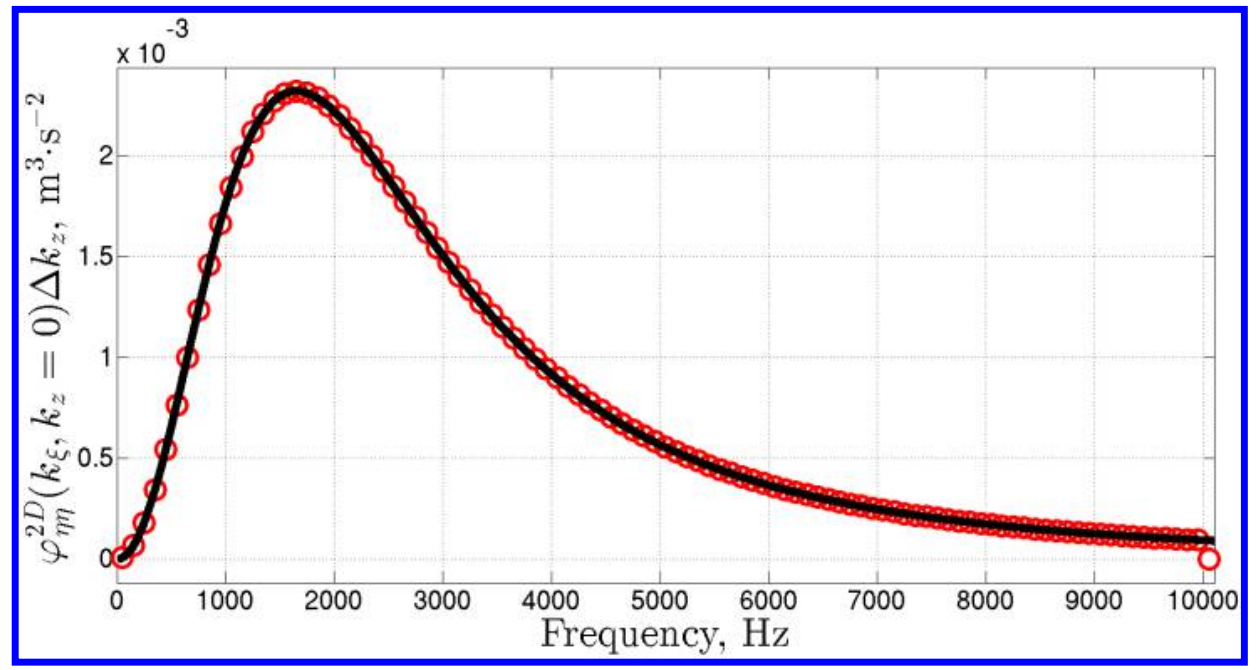

Fig. 5 Liepmann spectrum of the upwash velocity component (black solid line - ) and synthetic turbulence spectrum (red line with circles - ${ }^{-}$) obtained with $\Delta f=1 \mathrm{~Hz}$ and averaged over 250 blocks. 


\section{2. $\left(k_{1}, k_{3}\right)$ turbulence structure}

For $\left(k_{\xi}, k_{z}\right)$ turbulence, the same geometry and notations are used than previously as illustrated by Fig. 3 The only difference is that the wavenumber $k_{z}$ is no more equal to zero. Instead, the wavenumbers along the $z$ direction are discretized following $k_{z, n}=n \Delta k_{z}$. Now, $L \times N$ angular phases $\psi_{l n}$ are randomly selected. Fig. 6 validates the good implementation of this spanwise varying turbulence,

$$
u_{y}^{\prime}(\boldsymbol{X}, t)=\frac{2}{\cos \left(\beta_{c}\right)} \sum_{l=1}^{L} \sum_{n=-N}^{N} \sqrt{\varphi_{\eta \eta}^{2 D}\left(k_{\xi, l}, k_{z, n}\right) \Delta k_{\xi} \Delta k_{z}} \cos \left(k_{x, l} x+k_{z, n} z-\omega_{l} t+\psi_{l n}\right)
$$

even if the $k_{z}$ variation gives rise to statistical errors in the Power Spectral Density (PSD) spectrum.

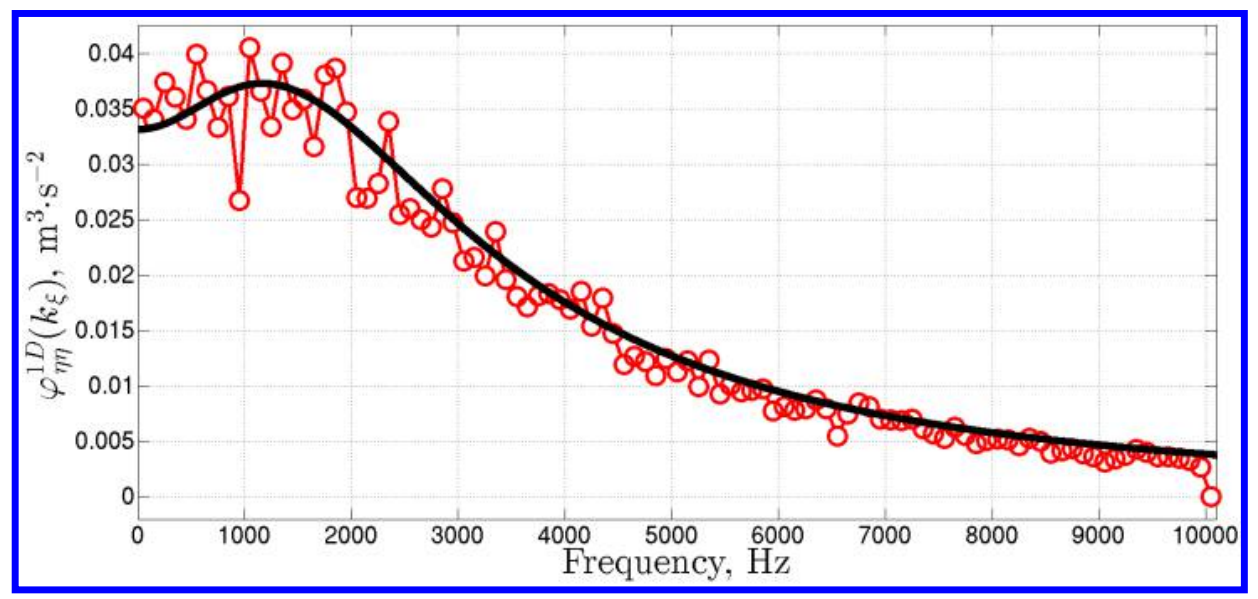

Fig. 6 Liepmann spectrum of the upwash velocity component (black solid line - ) and synthetic turbulence spectrum (red line with circles - ${ }^{-}$) obtained with $N=30, \Delta k_{z}=2 \pi / L_{z}, \Delta f=1 \mathrm{~Hz}$, and averaged over 250 blocks.

3. $\left(k_{1}, k_{2}, k_{3}=0\right)$ turbulence structure

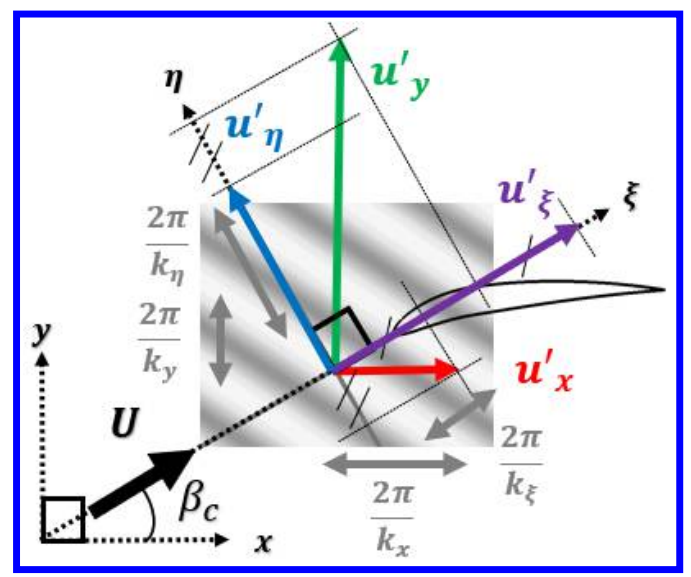

Fig. 7 Coordinate systems with associated wavenumbers and involved turbulent velocities for turbulence structures lying in the plane $\left(k_{1}, k_{2}, k_{3}=0\right)$.

The purpose of the structure $\left(k_{\xi}, k_{\eta}, 0\right)$, is to consider the pitchwise variations that should produce significant cascade effects in such rectilinear configurations. Fig. 7 is helpful to follow the discussion. There are several ways to derive the equations for the injected velocities $\left(u_{x}^{\prime}, u_{y}^{\prime}\right)$ or $\left(u_{\xi}^{\prime}, u_{\eta}^{\prime}\right)$ depending on the coordinate system. Two of them are listed below. Contrary to the $\left(k_{\xi}, k_{z}\right)$ turbulence for which the spectrum was integrated over $k_{\eta}$, the summation is 
explicitly defined here. Consequently, the $u_{\eta}^{\prime}$ component of the velocity is no more independent of the $k_{\eta}$ wavenumber. To ensure a divergence free solution, a second velocity component must be considered,

$$
\frac{\partial u_{\xi}^{\prime}}{\partial \xi}+\frac{\partial u_{\eta}^{\prime}}{\partial \eta}=0
$$

Consequently, $u_{\xi}^{\prime}$ is defined by $u_{\xi}^{\prime}=-k_{\eta} / k_{\xi} u_{\eta}^{\prime}$, and furthermore, $\varphi_{\xi \xi}^{3 D}\left(k_{\xi}, k_{\eta}, k_{z}=0\right)=\varphi_{\eta \eta}^{3 D}\left(k_{\xi}, k_{\eta}, k_{z}=0\right) k_{\eta}^{2} / k_{\xi}^{2}$. The spectrum along the $\xi$ axis is also correctly prescribed. Turbulence equations can also be obtained starting from the energy spectrum $E(k)$, as more usually considered in Stochastic Noise Generation and Radiation (SNGR) models [14],

$$
\boldsymbol{u}^{\prime}(\boldsymbol{X}, t)=2 \sum_{k=1}^{K} \sqrt{E\left(k_{k}^{\perp}\right) \Delta k^{\perp} \frac{2 \pi}{L_{z}}} \cos \left(\boldsymbol{k}_{\boldsymbol{k}}^{\perp} \cdot(\mathbf{X}-\mathbf{U} t)+\psi_{k}\right) \boldsymbol{\sigma}_{\boldsymbol{k}}
$$

where $k^{\perp}$ is defined such as $\boldsymbol{k}^{\perp} \cdot \boldsymbol{k}_{z}=0$ and $\boldsymbol{\sigma}$ is a unit vector such as $\boldsymbol{\sigma} \cdot \boldsymbol{k}^{\perp}=0$. Eq. (9) gives the discretized velocity for $K$ modes distributed over a disk. As for $\left(k_{\xi}, k_{z}=0\right)$ turbulence, a factor $2 \pi / L_{z}$ has to be taken into account to recover the expected magnitude of the spectra. However, here, to keep a simpler discretization in $\left(k_{\xi}, k_{\eta}\right)$ or $\left(k_{x}, k_{y}\right)$, with loops implemented in the generation routines, the turbulence modes are mapped on a rectangular box in the wavenumber space.

$$
\boldsymbol{u}^{\prime}(\boldsymbol{X}, t)=2 \sum_{l=1}^{L} \sum_{m=-M}^{M} \sqrt{\frac{E\left(\sqrt{k_{\xi, l}^{2}+k_{\eta, l m}^{2}}\right)}{\pi k} \Delta k_{\xi} \Delta k_{\eta} \frac{2 \pi}{L_{z}}} \cos \left(k_{\xi, l}(\xi-U t)+k_{\eta, l m} \eta+\psi_{l m}\right) \sigma_{l \boldsymbol{m}}
$$

A similar equation is provided by Gea-Aguilera et al. [7]. The wavenumber decomposition along the spatial directions in Eq. (10) easily ensures the periodic boundary condition along the $y$ direction by satisfying $\boldsymbol{k} \cdot \boldsymbol{y}=m 2 \pi / L_{y}$, with $m$ an integer. The formulation coming from the autocorrelation velocity spectra can be recovered considering that $E(k)=\varphi_{\eta \eta}^{3 D}\left(k_{\xi}, k_{\eta}, 0\right) 4 \pi k^{4} / k_{\xi}^{2}, E(k)=\varphi_{\xi \xi}^{3 D}\left(k_{\xi}, k_{\eta}, 0\right) 4 \pi k^{4} / k_{\eta}^{2}$ and that $\sigma_{\xi, l m}= \pm k_{\eta, l m} / k, \sigma_{\eta, l m}= \pm k_{\xi, l} / k$. The gusts are then generated as follows,

$$
\begin{aligned}
& u_{\xi}^{\prime}(X, t)=-2 \sum_{l=1}^{L} \sum_{m=-M}^{M} \frac{k_{\eta}}{k_{\xi}} \sqrt{\varphi_{\eta \eta}^{3 D}\left(k_{\xi, l}, k_{\eta, l m}, 0\right) \Delta k_{\xi} \Delta k_{\eta} \frac{2 \pi}{L_{z}}} \cos \left(k_{\xi, l}(\xi-U t)+k_{\eta, l m} \eta+\psi_{l m}\right) \\
& u_{\eta}^{\prime}(X, t)=2 \sum_{l=1}^{L} \sum_{m=-M}^{M} \sqrt{\varphi_{\eta \eta}^{3 D}\left(k_{\xi, l}, k_{\eta, l m}, 0\right) \Delta k_{\xi} \Delta k_{\eta} \frac{2 \pi}{L_{z}}} \cos \left(k_{\xi, l}(\xi-U t)+k_{\eta, l m} \eta+\psi_{l m}\right)
\end{aligned}
$$

Moreover, the wavenumbers in the upstream mean flow frame can be expressed as $k_{\xi, l}=\cos \left(\beta_{c}\right) k_{x, l m}+\sin \left(\beta_{c}\right) k_{y, m}$ and $k_{\eta, l m}=-\sin \left(\beta_{c}\right) k_{x, l m}+\cos \left(\beta_{c}\right) k_{y, m}$, which can be deduced from Fig. 7. The numerical implementation is validated for both streamwise and upwash velocity components as plotted in Fig. 8 . 


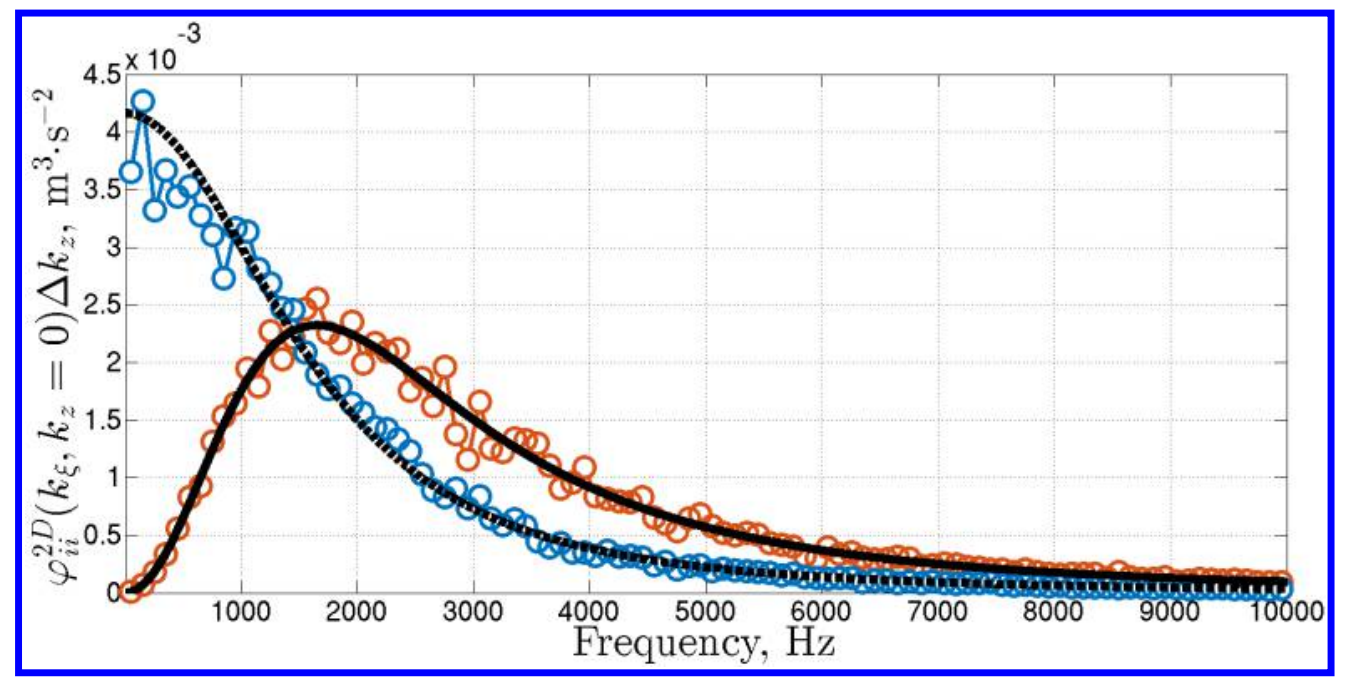

Fig. 8 Liepmann spectra of the upwash velocity component (black solid line - ) and the streamwise component (black dotted line .....). Synthetic turbulence spectra (of the upwash component in red line with circles - $\bigcirc$ - and streamwise component in blue line with circles - $\bigcirc-$ ) obtained with $M=16, L_{y}=s=8.5 \mathrm{~cm}$, $\Delta f=1 \mathrm{~Hz}$, and averaged over 250 blocks.

\section{A framework for generating fully 3D turbulence structures}

\section{Choice of an adapted energy spectrum description and equivalence with autocorrelation formulations}

Let us see now how to obtain a three (non-zero) wavenumber spectrum. This kind of turbulence structure will allow to investigate the role of all the wavenumbers on the aeroacoustic response for both the impinged baseline and serrated airfoils in a cascade configuration. In the first instance, wall boundary conditions are not considered. In order to obtain such a turbulence structure, several formulations could be considered a priori. Inconsistencies of ad-hoc formulations are clearly pointed out before reaching a suitable expression finally adopted in our method. In particular, it is demonstrated why a turbulence of the shape $\left(u_{\xi}^{\prime}\left(k_{\xi}, k_{\eta}, k_{z}\right), u_{\eta}^{\prime}\left(k_{\xi}, k_{\eta}, k_{z}\right)\right)$, defined as follows, is not usable in practice and why it raises the need for an additional component $u_{z}^{\prime}$.

$$
\begin{aligned}
& u_{\eta}^{\prime}(\boldsymbol{X}, t)=2 \sum_{l=1}^{L} \sum_{m=-M}^{M} \sum_{n=-N}^{N} \sqrt{\varphi_{\eta \eta}^{3 D}\left(k_{\xi, l}, k_{\eta, l m}, k_{z, n}\right) \Delta k_{\xi} \Delta k_{\eta} \Delta k_{z}} \cos \left(k_{\xi, l}(\xi-U t)+k_{\eta, l m} \eta+k_{z, n} z+\psi_{l m n}\right) \\
& u_{\xi}^{\prime}(\boldsymbol{X}, t)=-2 \sum_{l=1}^{L} \sum_{m=-M}^{M} \sum_{n=-N}^{N} \frac{k_{\eta, l m}}{k_{\xi, l}} \sqrt{\varphi_{\eta \eta}^{3 D}\left(k_{\xi, l}, k_{\eta, l m}, k_{z, n}\right) \Delta k_{\xi} \Delta k_{\eta} \Delta k_{z}} \cos \left(k_{\xi, l}(\xi-U t)+k_{\eta, l m} \eta+k_{z, n} z+\psi_{l m n}\right)
\end{aligned}
$$

The autocorrelation spectrum of the $u_{\xi}^{\prime}$ component where the summation over the $k_{\eta}$ wavenumber has been replaced by an integral is considered.

$$
\varphi_{\text {pseudo }, \xi \xi}^{2 D}\left(k_{\xi}, k_{z}\right)=\frac{\mathrm{TI}^{2} U^{2} L^{2}}{4 \pi} \frac{1}{k_{\xi}^{2}} \frac{\left(k_{\xi}^{2}+k_{z}^{2}\right)}{\left(1+L^{2}\left(k_{\xi}^{2}+k_{z}^{2}\right)\right)^{3 / 2}}
$$

After integration, Eq. (13) is providing a so-called "pseudo" 2D autocorrelation spectrum. The 1D spectrum should be obtained from an integration over $k_{z}$. However, this $\varphi_{\text {pseudo, } \xi \xi}^{2 D}\left(k_{\xi}, k_{z}\right)$ tends asymptotically to $1 /\left|k_{z}\right|$, which prevents the convergence of the integral with respect to $k_{z}$. Moreover, close to low frequencies the solution will diverge due to the $1 / k_{\xi}^{2}$ term. That is why, even if in practice $u_{\xi}^{\prime}$ is discretized over finite intervals and not over $\mathbb{R}^{2}$, this formulation leads to very high values of the velocity component along the $\xi$ axis. For a flat plate impacted by such gusts, there is no problem due to the fact that $u_{\xi}^{\prime}$ plays absolutely any role. However, for a NACA airfoil with a non zero thickness, 
an issue may appear if the magnitude of $u_{\xi}^{\prime}$ is much higher in comparison with what it should be if turbulence had been properly defined. Consequently, the generated gusts have to include a non-zero component $u_{z}^{\prime}$ in order to match correctly the three components of the autocorrelation spectra.

Each velocity component is now defined through its autocorrelation spectrum following Eq. (3). It is shown why it leads to an unsuitable form.

$$
u_{i}^{\prime}(\boldsymbol{X}, t)=2 \sum_{l=1}^{L} \sum_{m=-M}^{M} \sum_{n=-N}^{N} \sqrt{\varphi_{i i}^{3 D}\left(k_{\xi, l}, k_{\eta, l m}, k_{z, n}\right) \Delta k_{\xi} \Delta k_{\eta} \Delta k_{z}} \cos \left(k_{\xi, l}(\xi-U t)+k_{\eta, l m} \eta+k_{z, n} z+\psi_{l m n}\right)
$$

The divergence free equation, associated with Eq. (14), which has to be satisfied in order to avoid the creation of spurious sources can be written as:

$$
\frac{\partial u_{x}^{\prime}}{\partial x}+\frac{\partial u_{y}^{\prime}}{\partial y}+\frac{\partial u_{z}^{\prime}}{\partial z}=0 \Leftrightarrow \sum_{i} k_{i} \sqrt{\varphi_{i i}^{3 D}}=0
$$

When injecting $\varphi_{\xi \xi}^{3 D}$ and $\varphi_{\eta \eta}^{3 D}$ in Eq. (15), one can deduce that $\varphi_{z z}^{3 D}$ is inadequately prescribed.

$$
\varphi_{\text {pseudo, } z z}^{3 D}\left(k_{\xi}, k_{\eta}, k_{z}\right)=\frac{1}{k_{z}^{2}} \frac{\left(k_{\xi} \sqrt{k_{\eta}^{2}+k_{z}^{2}}+k_{\eta} \sqrt{k_{\xi}^{2}+k_{z}^{2}}\right)^{2}}{k_{\xi}^{2}+k_{\eta}^{2}} \varphi_{z z}^{3 D}\left(k_{\xi}, k_{\eta}, k_{z}\right)
$$

More precisely, it is weighted by a factor defined in Eq. (16) (supposing $k_{z} \neq 0$ and $\left|k_{\xi}\right|+\left|k_{\eta}\right| \neq 0$ ) in comparison with the correct spectrum. Consequently, the shape of the spectrum is strongly altered. Although the component $\varphi_{\eta \eta}$ plays the major role in the aeroacoustic response (as shown for example in [8]), non-negligible effects might be expected. For this reason, the most reasonable path to obtain a complete 3 non-zero wavenumber turbulence, relies again on the usual SNGR formalism already initiated with $\left(k_{1}, k_{2}, k_{3}=0\right)$ turbulence.

$$
\boldsymbol{u}^{\prime}(\boldsymbol{X}, t)=2 \sum_{k=1}^{K} \sqrt{E\left(k_{k}\right) \Delta k} \cos \left(\boldsymbol{k}_{\boldsymbol{k}} \cdot(\mathbf{X}-\mathbf{U} t)+\psi_{k}\right) \boldsymbol{\sigma}_{\boldsymbol{k}}
$$

The general form of Eq. (9) is given by Eq. (17). However, it is not possible to take directly into account through an energy spectrum formulation the periodic boundary conditions which should ensure that $k_{y}=m 2 \pi / L_{y}$. That is why, the approach proposed here is generalizing the key idea already probed with the $\left(k_{1}, k_{2}, 0\right)$ turbulence structure. The wavenumber space is no more discretized by spherical volumes of an equivalent radius $k$ and thickness $\Delta k$, but by rectangular boxes with an elementary volume $\Delta k_{\xi} \Delta k_{\eta} \Delta k_{z}$. The relation between the two discretizations is given by $\Delta k \cong \Delta k_{\xi} \Delta k_{\eta} \Delta k_{z} /\left(2 \pi k^{2}\right)$.

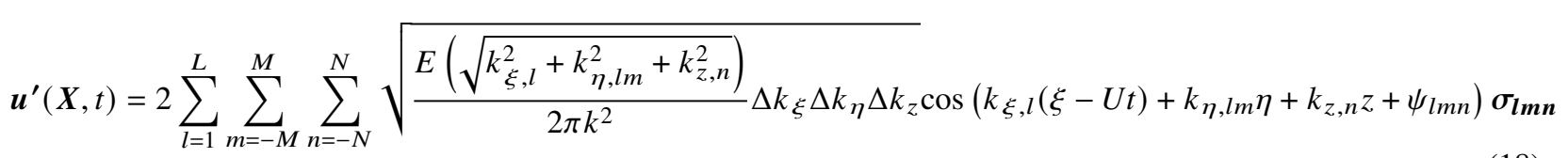

Hence, the modified energy spectrum formulation is now defined in the CAA frame by Eq. (18) in which the wavenumbers space is discretized over parallelepipeds in order to simplify the implementation and keep the framework used for previous formulations (using autocorrelation velocity spectra). A similar decomposition has been proposed by Gill et al. [15]. The divergence free condition of Eq. [17], is translated as: $\boldsymbol{k} \cdot \boldsymbol{\sigma}_{\boldsymbol{l m} \boldsymbol{n}}=0$, with $\boldsymbol{k}=\left(k_{\xi, l}, k_{\eta, l m}, k_{z, n}\right)^{T}$ and $\boldsymbol{\sigma}_{\boldsymbol{l m} \boldsymbol{n}}$, a unit vector. The direction of the turbulent velocity in Eq. (18) has to be determined. This is practically achieved by means of an efficient algorithm (similarly to [14]), which main steps are summarized in Appendix. A, and implemented inside the Fortran routines to generate the synthetic turbulence. In Appendix. B, a piece of demonstration is given to show the consistency between this modified energy spectrum formalism proposed here and previous formulations based on autocorrelation spectra. 


\section{Taking into account wall boundary conditions by means of a non-divergence free window function}

The full 3D turbulence formulation implies that a vertical velocity component $u_{z}^{\prime}$ has to be considered. This additional component has to be damped near the wall boundaries in order to avoid spurious noise sources, which is a non-tricky issue.

$$
\boldsymbol{u}^{\prime}(\boldsymbol{X}, t)=A f_{w}(z) \cos (\boldsymbol{k} \cdot \boldsymbol{X}-\omega t+\psi) \boldsymbol{\sigma}
$$

A window function $f_{w}(z)$ is applied to a given fluctuating velocity in Eq. [19], where $A$ is the initial amplitude of this given fluctuation, such as the velocity tends to zero near the wall boundaries. The chosen windowing, without care on divergence, is a gate function with half sinusoidal lobes on each side controlled by a length size $L_{w}$.

$$
\left\{\begin{array}{l}
\forall z \in\left[z_{\text {min }}, z_{\text {min }}+L_{w}\right], f_{w}(z)=\frac{1}{2}\left(1-\cos \left(\pi \frac{z-z_{\text {min }}}{L_{w}}\right)\right) \text { and } f_{w}^{\prime}(z)=\frac{\pi}{2 L_{w}} \sin \left(\pi \frac{z-z_{\text {min }}}{L_{w}}\right) \\
\forall z \in\left[z_{\text {min }}+L_{w}, z_{\text {max }}-L_{w}\right], f_{w}(z)=1 \text { and } f_{w}^{\prime}(z)=0 \\
\forall z \in\left[z_{\max }-L_{w}, z_{\max }\right], f_{w}(z)=\frac{1}{2}\left(1+\cos \left(\pi \frac{z-\left(z_{\max }-L_{w}\right)}{L_{w}}\right)\right) \text { and } f_{w}^{\prime}(z)=-\frac{\pi}{2 L_{w}} \sin \left(\pi \frac{z-\left(z_{\max }-L_{w}\right)}{L_{w}}\right)
\end{array}\right.
$$

The function $f_{w}$ proposed in Eq. 201 ensures a smooth transition at boundaries due to the fact that $f_{w}$ is $C^{1}$ everywhere on the interval $\left[z_{\min }, z_{\max }\right]$ and that $f_{w}\left(z_{\min }\right)=f_{w}\left(z_{\max }\right)=f_{w}^{\prime}\left(z_{\min }\right)=f_{w}^{\prime}\left(z_{\max }\right)=0$.

$$
\operatorname{div}\left(\boldsymbol{u}^{\prime}\right)=f_{w}^{\prime}(z) A \cos (\boldsymbol{k} \cdot \boldsymbol{X}-\omega t+\psi) \sigma_{z} \neq 0
$$

However, this window function implies that the divergence free property of the synthetic turbulence field is no more ensured as shown by Eq. 21]. The impact on the far-field acoustics will be discussed on the baseline numerical simulations in section $\mathrm{V}$.

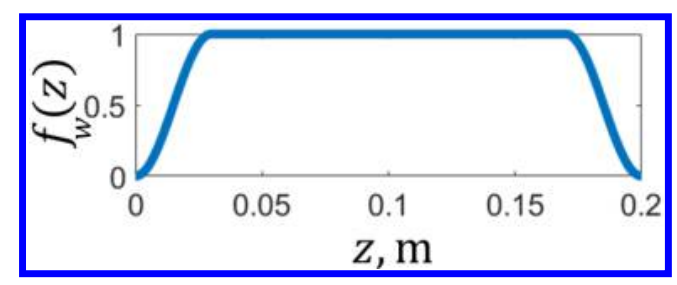

Fig. $9 f_{w}(z)$ with $R_{w}=15 \%$ and $L_{z}=0.2 \mathrm{~m}$.

An example of a window function is presented in Fig. 9 (where $R_{w}=L_{w} / L_{z}=15 \%$ ). Figs. 10a and $10 \mathrm{~b}$ show respectively turbulence spectra at $z=0\left(\right.$ where $\left.f_{w}(z)=1\right)$ and $z=0.185\left(\right.$ where $\left.f_{w}(z)=0.5\right)$. They demonstrate a good agreement with the theoretical spectra, which validates the adapted formulation relying on the energy spectrum. Since the windowing consists simply in the multiplication of the turbulence fluctuations by a constant, the spectra amplitude at $z=0.185$ are simply reduced here by a factor $0.5^{2}$ as illustrated in Fig. $10 \mathrm{~b}$ 


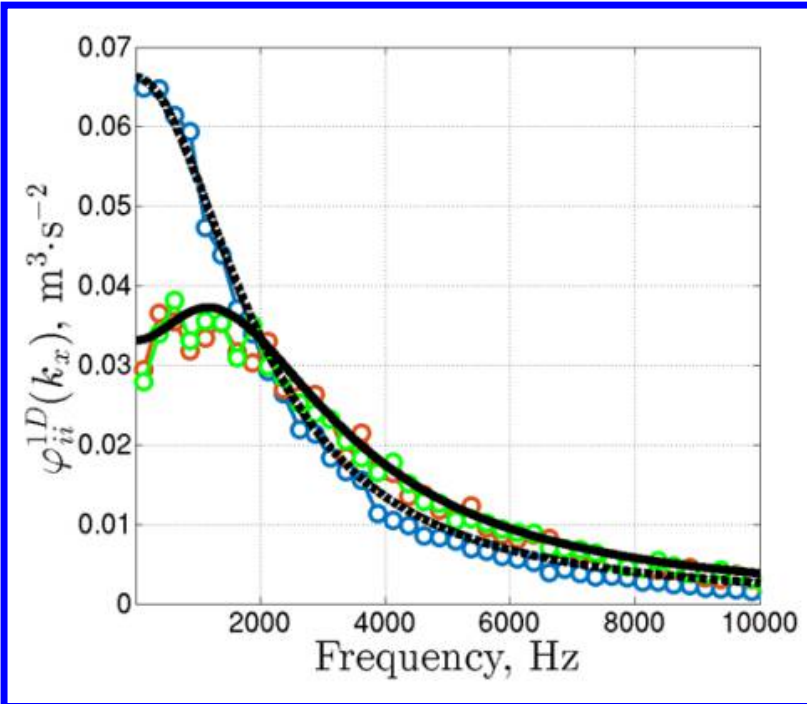

a)

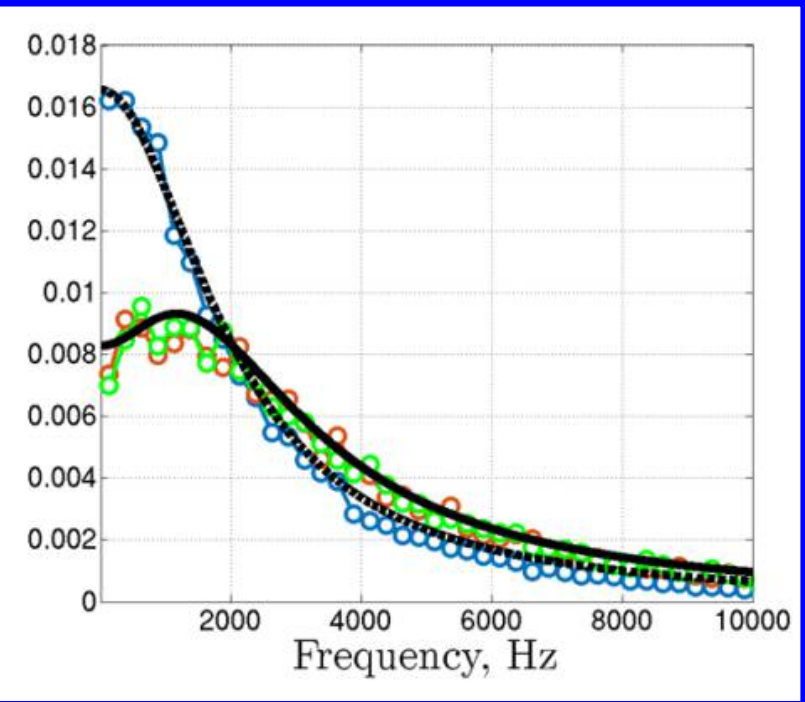

b)

Fig. 10 Liepmann spectra of the upwash and spanwise velocity components (black solid line streamwise component (black dotted line .....). Synthetic turbulence spectra in solid line with circles (upwash component in red - $\bigcirc-$, spanwise component in green - $\bigcirc_{-}$, and streamwise component in blue - $\bigcirc-$-) obtained with $N=30, R_{w}=15 \%, M=16, L_{y}=s=8.5 \mathrm{~cm}, \Delta f=1 \mathrm{~Hz}$, and averaged over 250 blocks. Spectra from the left figure obtained for location $z=0.1$ and from the right figure at location $z=0.185$ where $f_{w}(z)=0.5$.

\section{Theoretical development and implementation of a divergence free window function}

Equation (21) shows that in order to obtain a divergence free formulation, the expressions governing the velocity have to be modified. The choice has been made to only modify the component $u_{z}^{\prime}$ since it plays a minor role in the noise source generation process.

$$
\frac{\partial u_{z}^{\prime}}{\partial z}=-f_{w}(z) A \sin (\boldsymbol{k} \cdot \boldsymbol{X}-\omega t+\psi) k_{z} \sigma_{z}+f_{w}^{\prime}(z) A \cos (\boldsymbol{k} \cdot \boldsymbol{X}-\omega t+\psi) \sigma_{z}-g_{w}^{\prime}(z) A \sigma_{z}
$$

Consequently, in order to cancel the additional spurious term in Eq. (21) the derivative of $u_{z}^{\prime}$ given by Eq. (22) is modified by means of a correction function $g_{w}(z)$. After some calculations (ensuring $\left(f_{w}, g_{w}\right) \in C^{1}$ and boundary conditions) presented in Appendix. C, one can show that the following equality has to be satisfied.

$\cos \left(k_{x} x+k_{y} y+k_{z} z_{\min }+\frac{k_{z} L_{w}}{2}-\omega t+\psi\right) \cos \left(\frac{k_{z} L_{w}}{2}\right)=\cos \left(k_{x} x+k_{y} y+k_{z} z_{\min }+k_{z} L_{z}-\frac{k_{z} L_{w}}{2}-\omega t+\psi\right) \cos \left(\frac{k_{z} L_{w}}{2}\right)$

Two cases arise from Eq. 23). Firstly, let us consider that $\cos \left(k_{z} L_{z} / 2\right)=0$ which leads to $k_{z}=(2 n+1) \pi / L_{w}$ with $n \in \mathbb{N}$. However, since $k_{z} \neq \pi / L_{w}$ (see Appendix. B), this solution implies that the discretization can not be uniformly spaced. Moreover, if $L_{w}$ is chosen too small the discretization steps tends to become very wide which could affect the precision of the solution. The another possibility is to consider $k_{z}=2 n \pi /\left(L_{z}-L_{w}\right)$. The case $R_{w}=15 \%$, which gives the same envelope that for the non divergence-free scenario, but with a slightly shifted discretization of the $k_{z}$ wavenumbers, is considered. The generated spectra with the new implementation are compared with the analytical solutions in Figs. 11a and 11b There is a good agreement for the streamwise and upwash components for all cases. The fact that the spanwise spectra are altered is related to the choice of applying the correction on the $u_{z}^{\prime}$ component of the velocity. 


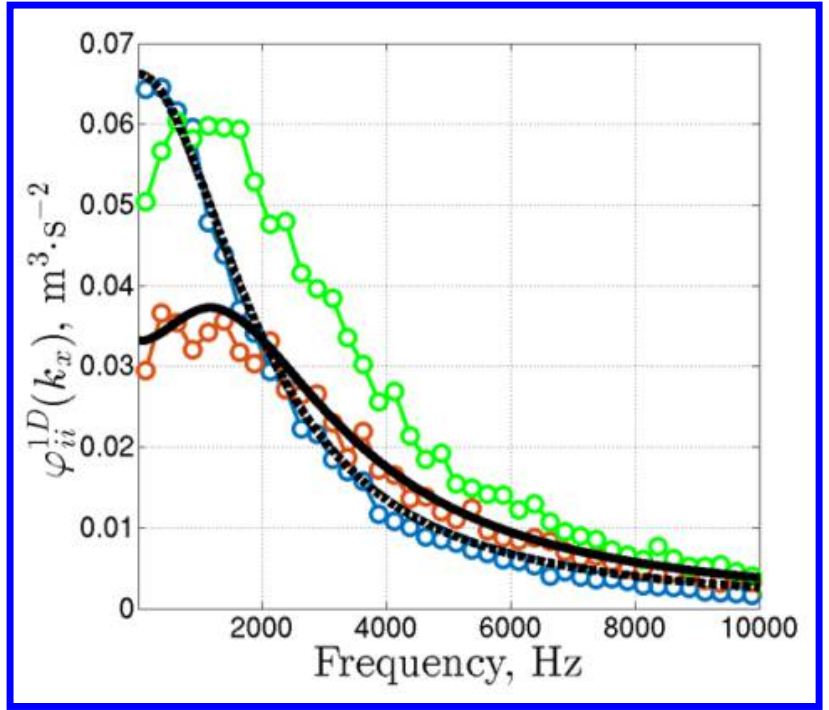

a)

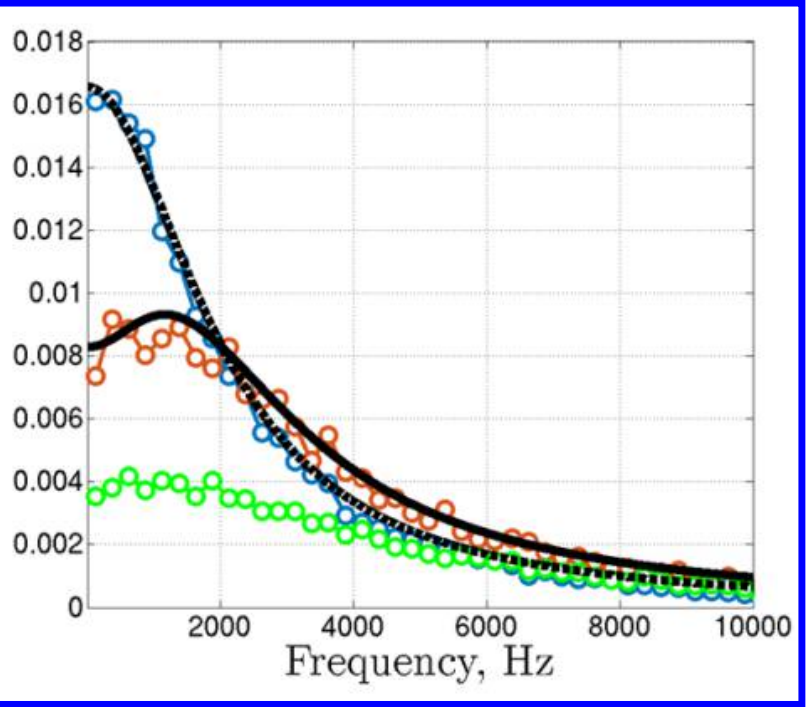

b)

Fig. 11 Liepmann spectra of the upwash and spanwise velocity components (black solid line - ) and the streamwise component (black dotted line -....). Synthetic turbulence spectra with divergence free formulation in solid line with circles (upwash component in red - $\bigcirc-$, spanwise component in green - $\bigcirc-$, and streamwise component in blue - $\bigcirc-$ ) obtained with $N=30, R_{w}=15 \%, M=16, L_{y}=s=8.5 \mathrm{~cm}, \Delta f=1 \mathrm{~Hz}$, and averaged over 250 blocks. Spectra from the left figure obtained for location $z=0.1$ and from the right figure at location $z=0.185$ where $f_{w}(z)=0.5$.

\section{Some words about the numerical optimization}

The synthetic turbulence generation process has been implemented in Fortran code. Previous routines used at ONERA for annular geometries [1, 2], have been rewritten in a Cartesian coordinate system. In parallel, several features have been added in order to lower the CPU generation cost. Turbulence structures having a symmetry axis are generated only on a line of the mesh and duplicated afterwards on all the injection plane. Turbulence equations have been decomposed as much as possible using trigonometric identities to factorize operations. In order to speed-up the generation process of a full 3D synthetic turbulence some additional optimization has been needed. It has been chosen not to generate turbulence over all the time steps of the simulation similarly to [11]. The missing time steps are recovered by a linear interpolation. For example, here, the synthetic turbulence field has been generated one time step over 10 on the baseline computations, still ensuring 40 points per period at the highest simulated frequency. For each 3 channels simulation presented below, the 3D turbulence generation process has required only around 10 hours on one thousand cores, for which the CAA converged solution was obtained in about 30 hours on 1077 cores.

\section{Aeroacoustic applications on the rectilinear cascade configuration}

\section{A. Introduction to the numerical set-up on the baseline (untreated) geometry}

\section{Inviscid mean flow calculation}

The mean flow has been computed by means of a 2D open source Euler code proposed in [25]. Inflow conditions are indicated in Fig. 12. A few loops have been performed to get the targeted upstream Mach number of 0.3. This has been done by adjusting the exhaust static pressure. This approach was shown to be an efficient alternative to previous RANS approach which requires some corrections near the solid boundaries in order to remove boundary layers and recirculation zones, incompatible with the inviscid assumption of the CAA code. For the mean flow computation, a single channel simulation with periodic boundary condition has been considered. The obtained mean velocity field has been then duplicated and interpolated on the CAA mesh for acoustic multi-channel computations. It has to be noted that the upstream Mach number and the entrance flow angle used for the CAA have been chosen before the final values (see 
Table 11 have been provided by the consortium. However, it should not modify the conclusions drawn here.

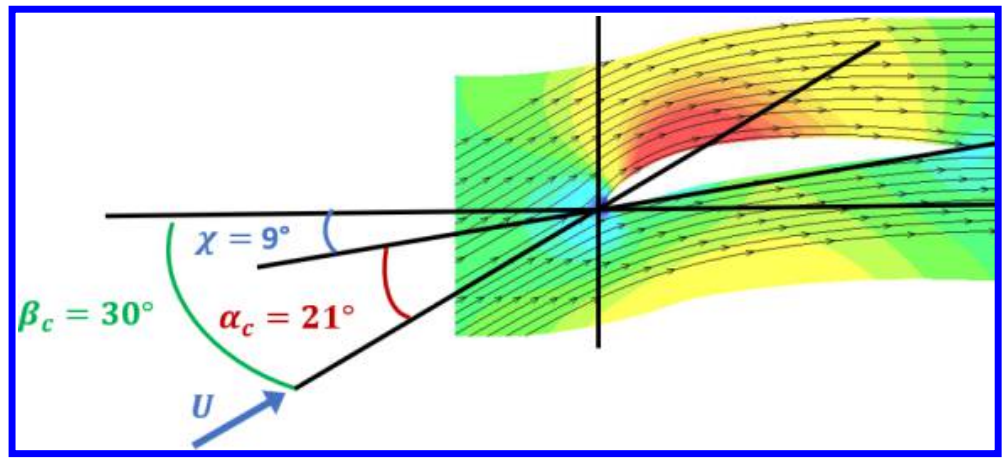

Fig. $12 U_{x}$, levels between 60 and $120 \mathrm{~m} \cdot \mathrm{s}^{-1}$.

\section{Simulated configuration and main assumptions of the aeroacoustic computations}

The CAA simulation setup uses the boundary conditions presented in Figs. 3 and 12 . Except for the one channel computations and the parametric study on the vane count from [5] on the baseline geometry, which are performed on a $\mathrm{H}$ pattern grid (more discussed in [5]), all the CAA grid is designed using an $\mathrm{O}-\mathrm{H}$ pattern plotted in Fig. 14] This choice has been made in order to facilitate the generation of the 3D mesh for the serrated geometry. Indeed, the mesh is practically designed using an in-house tool ersat $Z$ which allows to apply suitable $3 \mathrm{D}$ deformations on the reference skeleton to obtain the serrated shape and to extend them within the grid volume. For more information about the meshes and the preliminary results one may refer to the previous paper from Buszyk et al. [5]. The main conclusion from this preliminary study was that multi-channel computations are mandatory to avoid spurious resonance phenomena in the mid frequency range resulting from periodic boundary conditions and requiring to include at least 3-vane channels in the CAA domain. A typical result from these parametric studies [5] is shown in Fig. 13. All the following simulations discussed in the next paragraphs are achieved using 3-vane channels and first solutions from [5] are completed using full 3D turbulence modeling.

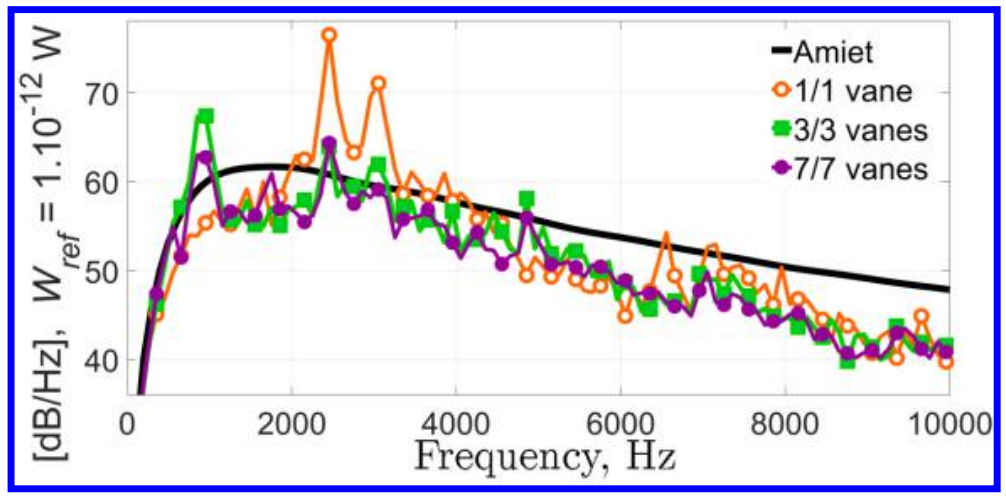

Fig. 13 Downstream sound power per vane. Parametrical study on the number of vanes (channels) in the CAA domain taken from [5], with a $\left(k_{\xi}, k_{\eta}, k_{z}=0\right)$ turbulence. Computations performed with $\Delta f=10 \mathbf{H z}$ and averaged over 10 blocks. 


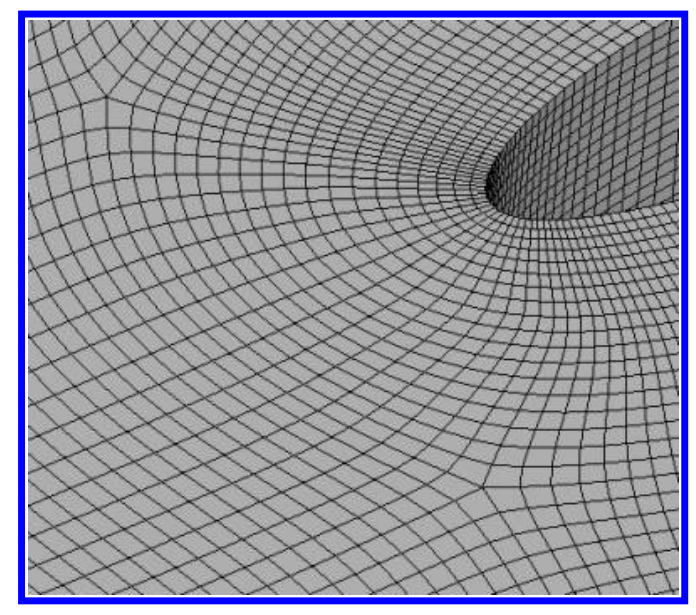

Fig. 14 O-H pattern CAA mesh.

\section{B. Aeroacoustic predictions on the baseline (untreated) geometry}

The acoustic computations with a 3D turbulence injection are performed using 3-vane channels which is also a good compromise in terms of CPU [5]. The parameters used for the generation of the 3D synthetic turbulence are the following: $L_{y}=0.255 \mathrm{~m}, M=48, N=30$, and $\Delta f=100 \mathrm{~Hz}$. In Fig. 15. snapshots of synthetic turbulent flows related to the transverse velocity $\left(u_{y}^{\prime}\right)$ are clearly showing the different patterns issued from 1D (a), 2D (b) and (c), and 3D (d) injected turbulence. Note that 1D (parallel gust), 2D (planar), and full 3D structures are respectively linked to the number of velocity components $(1,2$ or 3$)$ [15] and then to the non-zero wavenumbers involved in the present turbulence models. For the latter 3D turbulence, the velocity field has been cropped in order to avoid the visual effect induced by the window $f_{w}(z)$ at the borders.

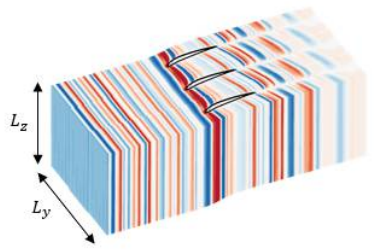

a)

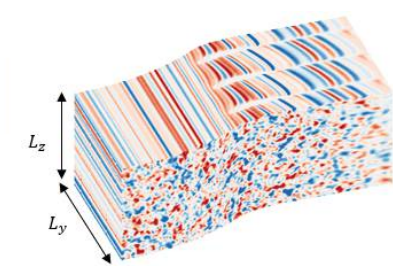

b)

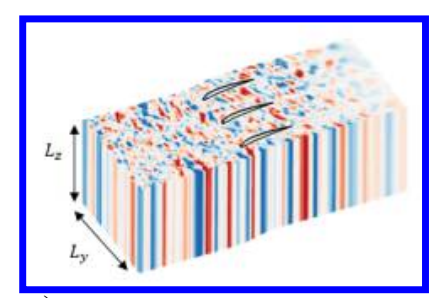

c)

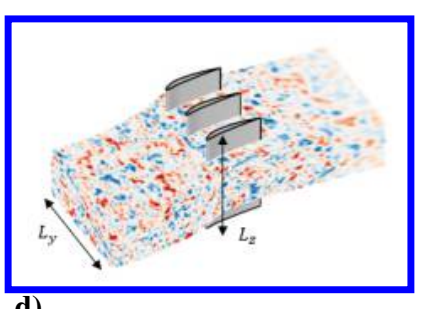

d)

Fig. 15 Turbulent-like velocity snapshots for $u_{y}^{\prime}$ (levels between $\pm 2 \mathbf{m} \cdot \mathbf{s}^{-1}$ for the $k_{z}=0$ cases and between $\pm 10 \mathbf{m} \cdot \mathbf{s}^{-1}$ otherwise). Turbulence structures from (a) to (d): $\left(k_{\xi}, k_{z}=0\right),\left(k_{\xi}, k_{z}\right),\left(k_{\xi}, k_{\eta}, k_{z}=0\right)$, and $\left(k_{\xi}, k_{\eta}, k_{z}\right)$.

The pressure fluctuations are then extracted at the vane skin and radiated in the far-field using an in-house code (MIA). MIA solves the FWH equation restricted here to the loading noise term with a solid surface formulation. The power spectra were obtained by a weighted angular integral over half a circle downstream of the cascade, as performed in [5]. In order to compensate the lack of energy input due to the window function $f_{w}(z)$ (with $R_{w}=0.15$ ), a simple correction has been applied to the spectra: $20 \log \left(1 /\left(1-R_{w}\right)\right)$. The numerical spectra are compared to Amiet's solution for an isolated flat plate in Fig. 16. Please note that a cascade model as proposed in [28-30] might be used to get a reliable reference solution, so that the Amiet-based spectrum is only giving a biased estimate by neglecting the cascade effect (quite significant in this configuration). In high frequencies, Amiet's model is overpredicting the acoustic spectra because it does not take into account any thickness. For $\left(k_{\xi}, k_{z}=0\right),\left(k_{\xi}, k_{z}\right)$, and $\left(k_{\xi}, k_{\eta}, k_{z}\right)$ turbulent structures, the computations have been performed with $\Delta f=100 \mathrm{~Hz}$ and with $\Delta f=20 \mathrm{~Hz}$ for $\left(k_{\xi}, k_{\eta}, k_{z}=0\right)$ turbulence. 


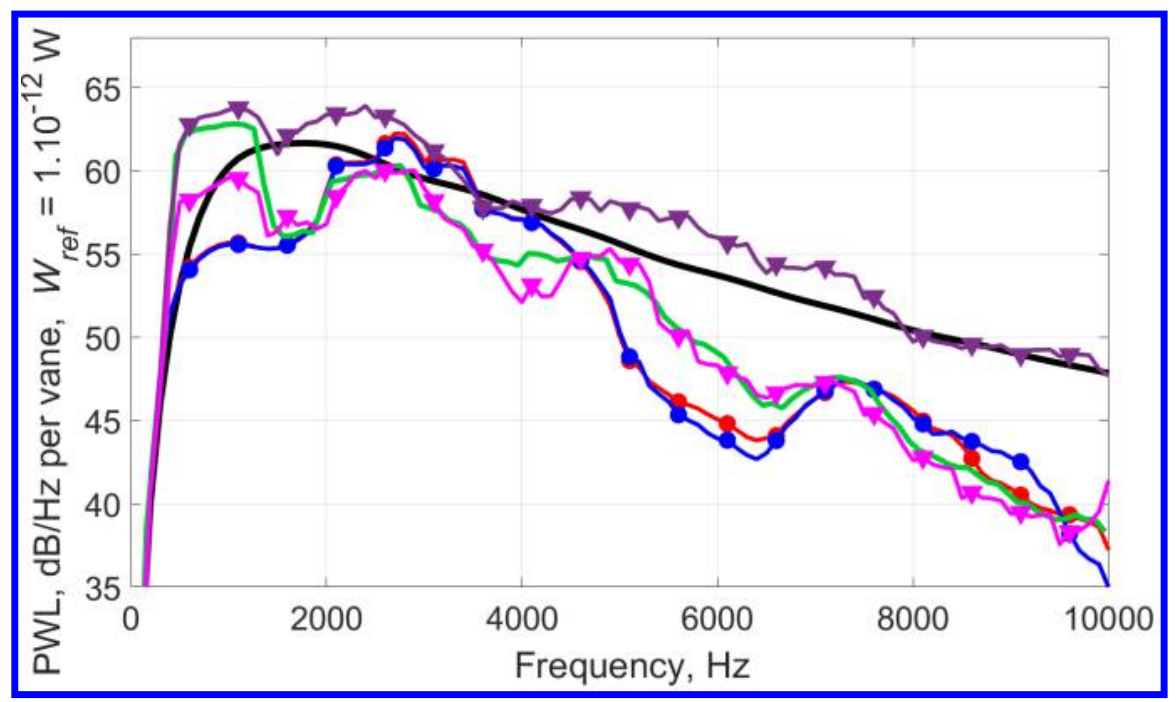

Fig. 16 Downstream sound power per vane. Amiet's solution for an isolated flat plate in black solid line - Numerical solutions for different synthetic turbulence injected in 3-channels computations in color: $\rightarrow$ $\left(k_{\xi}, k_{z}=0\right), \rightarrow\left(k_{\xi}, k_{z}\right), \longrightarrow\left(k_{\xi}, k_{\eta}, k_{z}=0\right), \neg\left(k_{\xi}, k_{\eta}, k_{z}\right)$ with $\operatorname{div}\left(\boldsymbol{u}^{\prime}\right) \neq 0$, and $\rightarrow\left(k_{\xi}, k_{\eta}, k_{z}\right)$ with $\operatorname{div}\left(u^{\prime}\right)=0$. A $1 \mathrm{kHz}$ moving average has been applied to raw CAA spectra.

Regarding the numerical spectra associated with $\left(k_{\xi}, k_{z}=0\right)$ and $\left(k_{\xi}, k_{\eta}, k_{z}=0\right)$ turbulence, one may notice that taking into account $k_{\eta}$ provides a more linear shape with respect to the attenuation slope in medium and high frequency range. However, the bump (around $800 \mathrm{~Hz}$ ) due to a numerical amplification using periodicity conditions over 3-vane channels instead of 7 is stronger when a turbulence with a varying $k_{\eta}$ is injected, see Fig. 15 from [5]. Regarding the comparison between CAA results and Amiet's solution, the levels are definitely over-estimated by the isolated flat plate approximation. This is why, even if the non-zero divergence turbulence is the closest solution to one from Amiet, it represents in fact the worst numerical prediction. Indeed, following literature [30, 31], a few decibels reduction is at least expected in low frequencies due to the cascade effect and in high frequencies due to the non-zero thickness of the airfoils [32]. Looking at the divergence free result, taking into account the $k_{z}$ wavenumber seems not to modify the spectra (green vs. pink curves), which is in accordance with previous numerical simulations (in [5]) and with Amiet's theory.

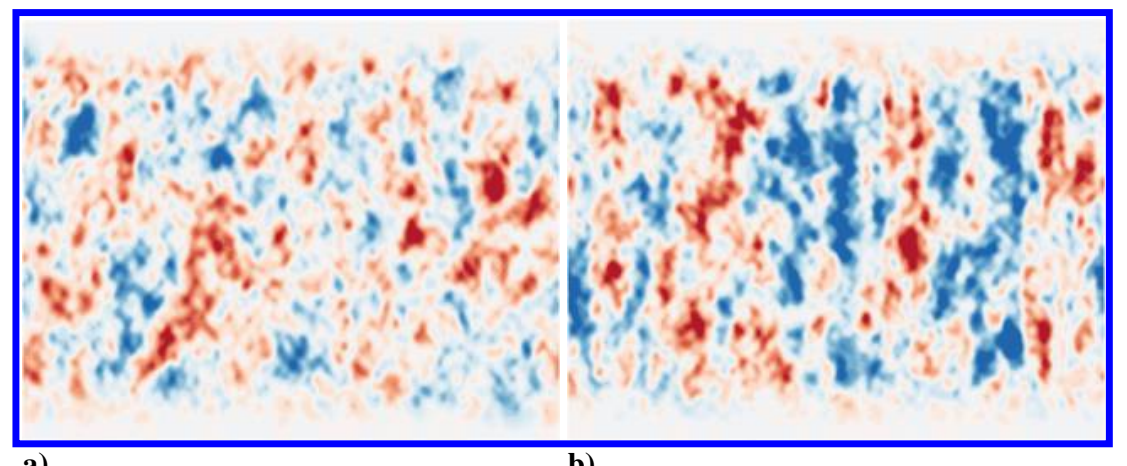

a)

b)

Fig. $17 u_{z}^{\prime}$ (levels between $\pm 10 \mathbf{m} \cdot \mathbf{s}^{-1}$ ) at the injection surface in the $(y, z)$ plane. Fully 3D turbulence with non-zero divergence (on the left) and with divergence free formulation (on the right). 


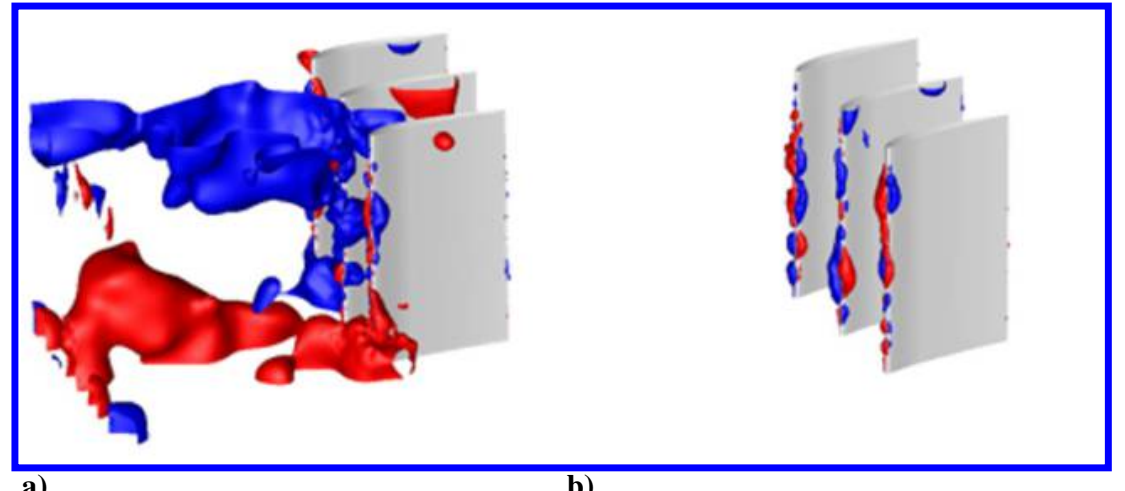

a)

b)

Fig. 18 Isosurfaces of $p^{\prime}$ at \pm 200 Pa. Turbulence $\left(k_{\xi}, k_{\eta}, k_{z}\right)$ with non-zero divergence (on the left) and $\left(k_{\xi}, k_{\eta}, k_{z}\right)$ with divergence free formulation (on the right).

Snapshots of the turbulent-like velocity $u_{z}^{\prime}$ at the injection plane are plotted in Fig. 17 The structure with the divergence-free condition (Fig. 17b) appears to be less homogeneous compared with those obtained without the correction term on $u_{z}^{\prime}$ (Fig. 17a, which is in accordance with the deviations on autocorrelation spectra presented in Fig. 11 However, as clearly visible on iso-surfaces of fluctuating pressure in Fig. 18, the non-zero divergence formulations (Fig. 18a gives rise to local intense spots extending through the CAA domain and interacting with the vanes, and responsible for some noise increase. On the contrary, the solution obtained with the divergence-free formulation (Fig. $18 \mathrm{~b}$ is found to be much cleaner without generation of additional numerical spurious sources.

\section{Design of a passive treatment based on leading edge serrations}

The leading edge serrations have been firstly designed on a 2D plane, plotted in Fig. 19a following the background from previous studies, the most recent is presented in [2]. The theoretical optimal serration wavelength, defined from [19], corresponds to twice the turbulence length scale of $8 \mathrm{~mm}$. The serration amplitude is defined directly related to the serration angle and so the ratio $h_{s} / \lambda_{s}$. Although the acoustic benefit could be improved by increasing $h_{s}$, a practical limitation of the ratio $h_{s} / c$ is required to avoid a noticeable loss of aerodynamic performances for industrial applications. Such a threshold has been used in [2]. Hence, setting $h_{s} / \lambda_{s}=1$ can be considered as a reasonable value close to the optimum design. For the numerical computation, in order to get an integer number of serrations along the span, the quantities have been slightly increased to end with $h_{s}=\lambda_{s}=16.7 \mathrm{~mm}$. To apply the planar deformation on the 3D geometry of the airfoil, the ersatZ tool has been used. The first step has consisted in the extraction of the camber and thickness laws. An homothety has been then applied to these laws in order to obtain the shape of the airfoil at the roots of the serrations. To get the airfoil geometry at the hills of the serrations, the camber law was extended at the leading edge, keeping a constant angle as illustrated in Fig. 19b. The ersatZ modeler which is able to extend the skeleton deformations (see Fig. 19c) to the cells of the volume mesh has been used to apply a morphing of the baseline CAA grid, presented in Fig. 20, in order to perform aerocoustic simulations on the treated design. 


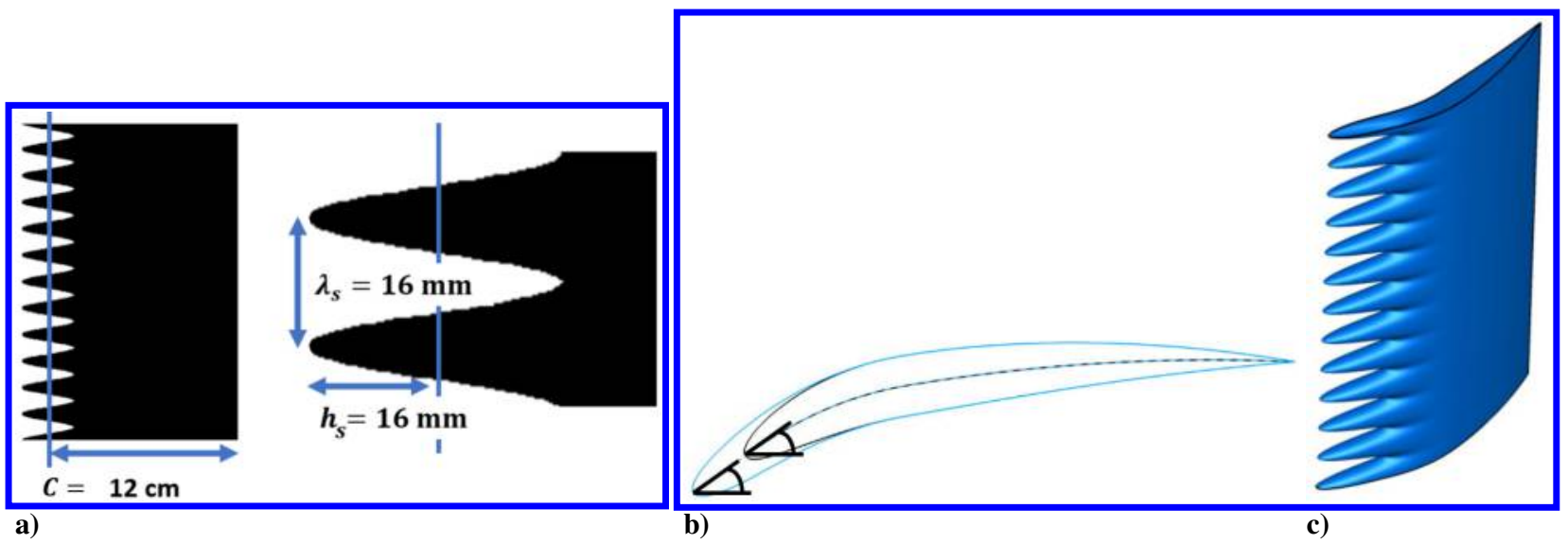

Fig. 19 2D plot of the serration geometry (on the left). Diagram of the deformation applied on the NACA 7310 airfoil (on the middle), and 3D geometry obtained by ersatzZ (on the right).

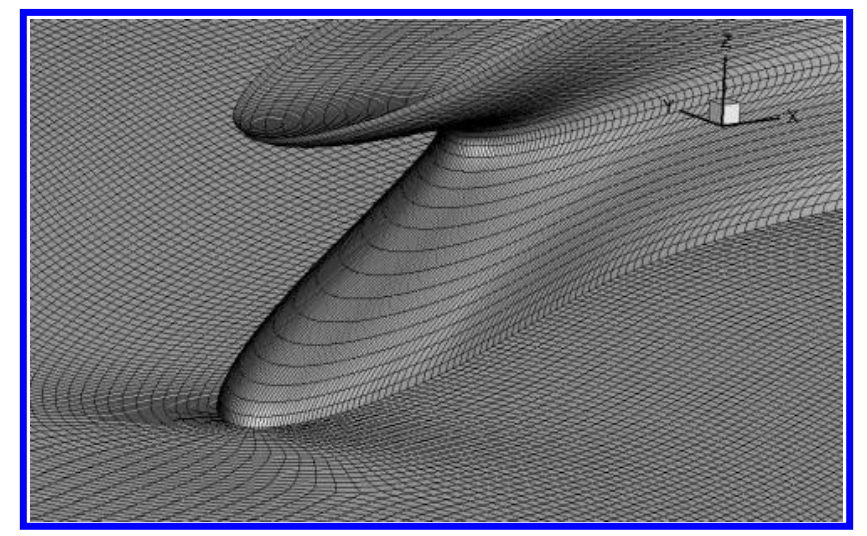

Fig. 20 Views of the skin CAA mesh and $z$ slice at the peak of the serration.

\section{Assessment of the noise reduction provided by the serrated geometry}

\section{Mean flow and CAA computation}

In order to obtain the mean flow around the new geometry with the 2D Euler open-source code, the computation was performed on $z$ planes, from which the velocity field has been then interpolated on the $3 \mathrm{D}$ CAA mesh. Thus, $U_{z}$ is set equal to zero in the domain, which is a proper approximation. Indeed, the vertical (radial) component of the mean flow velocity has shown to be almost negligible in comparison with the other velocity components by RANS solutions discussed in Section VI, and also consistent with previous studies on realistic turbofan configurations [2]. The resulting Euler mean flow fields, in terms of axial velocity maps and streamlines visualization, are plotted in Figs. 21a and $21 b$ respectively for planar cuts at root and peak of the serration. The streamlines are perfectly aligned to the airfoil geometry for these two tricky positions, which should allow to properly assess the turbulence-airfoil sources and sound propagation in the CAA. 


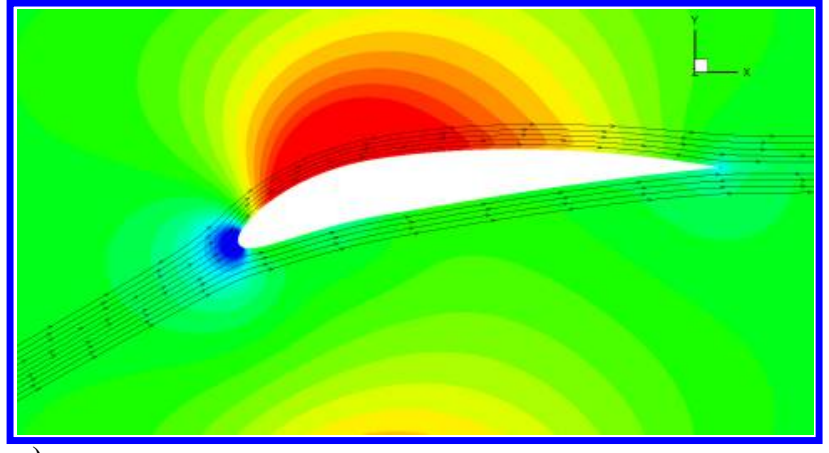

a)

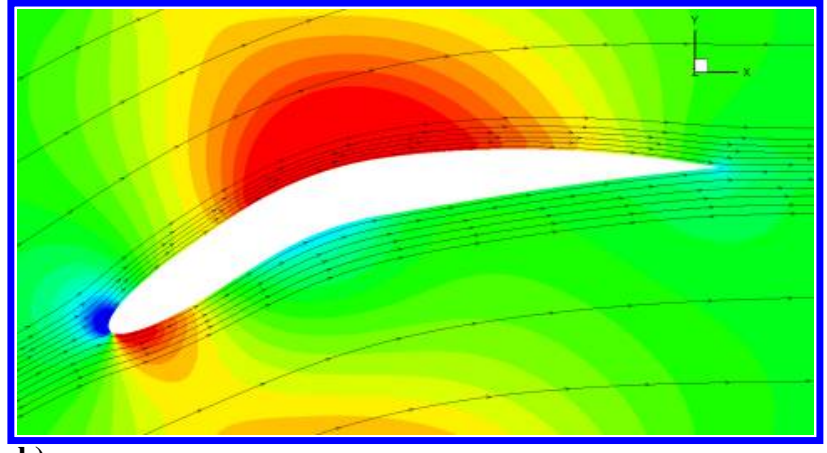

b)

Fig. 21 Isocontour maps of $U_{x}$ (levels between 60 and $120 \mathrm{~m} \cdot \mathrm{s}^{-1}$ ) with streamlines. Cuts at the root (left) and peak (right) of the serration.

The $z$ vorticity isosurfaces issued from the fully 3D turbulence simulation on the serrated case are plotted in Fig. 22. in which the contour maps of the RMS pressure fluctuations on the vane skin are surimposed. Moreover, the strongest pressure sources are located at the roots and peaks of the serrations in accordance with the literature [2, 8]. Finally, the pressure fluctuations are equally distributed over the 3 vanes, which satisfies the periodicity conditions (enforced in the transverse direction) and indicates the good convergence of the CAA computation.

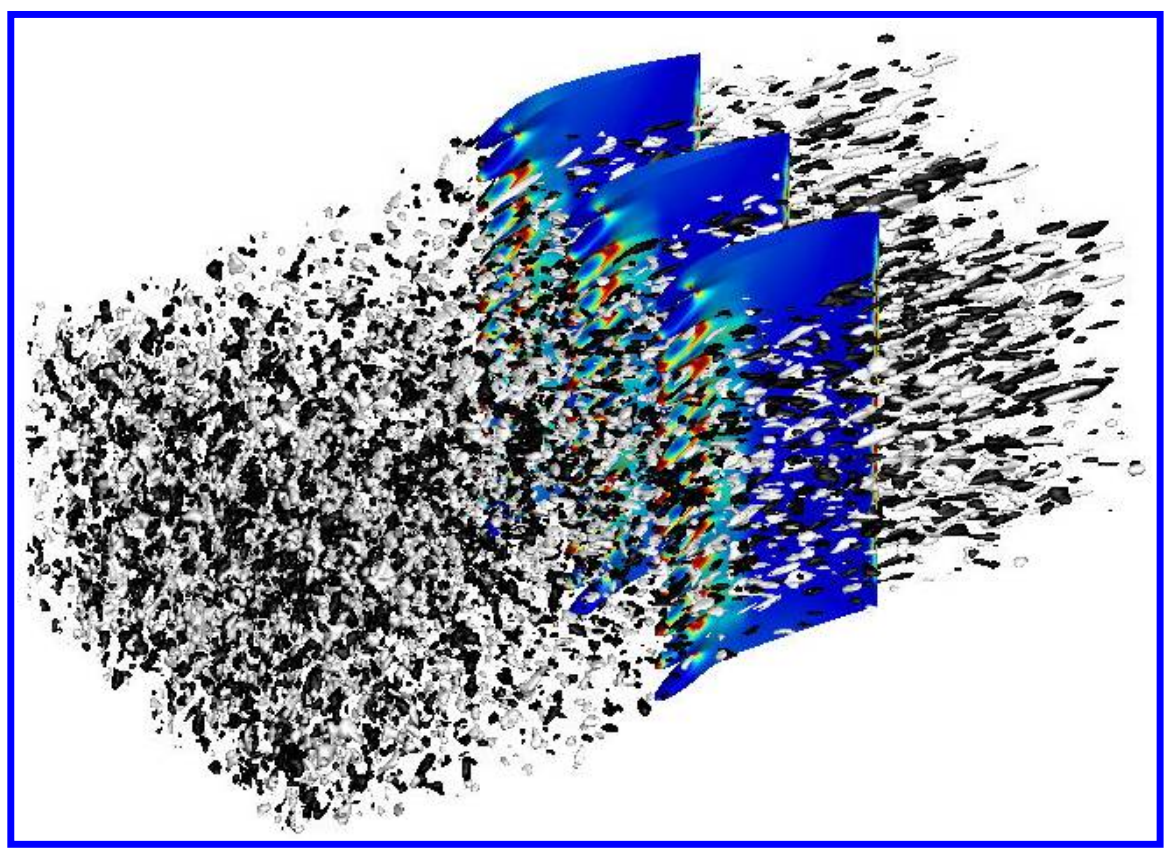

Fig. 22 Isosurfaces of vorticity $\omega_{z}^{\prime}, \mathbf{s}^{-1}$ and RMS wall pressure $p_{r m s}^{\prime}$, Pa (levels between 0 and 250 Pa).

\section{Sound Power Level reductions $(\triangle P W L)$ from CAA and comparison with analytical and empirical solutions}

The noise reduction assessed in terms of $\triangle$ PWL spectra are summarized in Fig. 23 . The numerical predictions with the different synthetic turbulence injections are compared with both an analytical solution and a semi empirical law proposed by ISVR [19]. The analytical solution presented in [20] solves the Helmholtz advective equation using the Wiener-Hopf (WH) technique for any piecewise leading edge geometries. It has been slightly modified for applications to finite span airfoils in [2, 5]. The semi-empirical law (from [19]) provides the noise optimal noise reduction (occurring when $\lambda_{s}=2 L_{t}$ ) for a single-wavelength geometry in terms of the Strouhal number: $\Delta \mathrm{PWL}=10 \log (S t)+10$. There is a good agreement between the different solutions in the medium frequency range especially in comparison with the semi-empirical law. As discussed in previous numerical studies [5, 27], ignoring the spanwise turbulence variation leads 
to an overprediction of the noise reduction particularly in high frequencies. The 3D turbulence numerical prediction (in pink) exhibits a slightly smaller noise reduction in high frequencies compared to other CAA computations in particular the 2-wavenumbers $\left(k_{\xi}, k_{z}\right)$ formulation (in blue). Practically, this study tends to show that the $\left(k_{\xi}, k_{z}\right)$ turbulence model is a good compromise in terms of accuracy vs. CPU time. Moreover, the $\triangle \mathrm{PWL}$ spectra achieved in this case is found to be almost identical when using 1-channel or 3-channels simulations, which makes this approach very attractive.

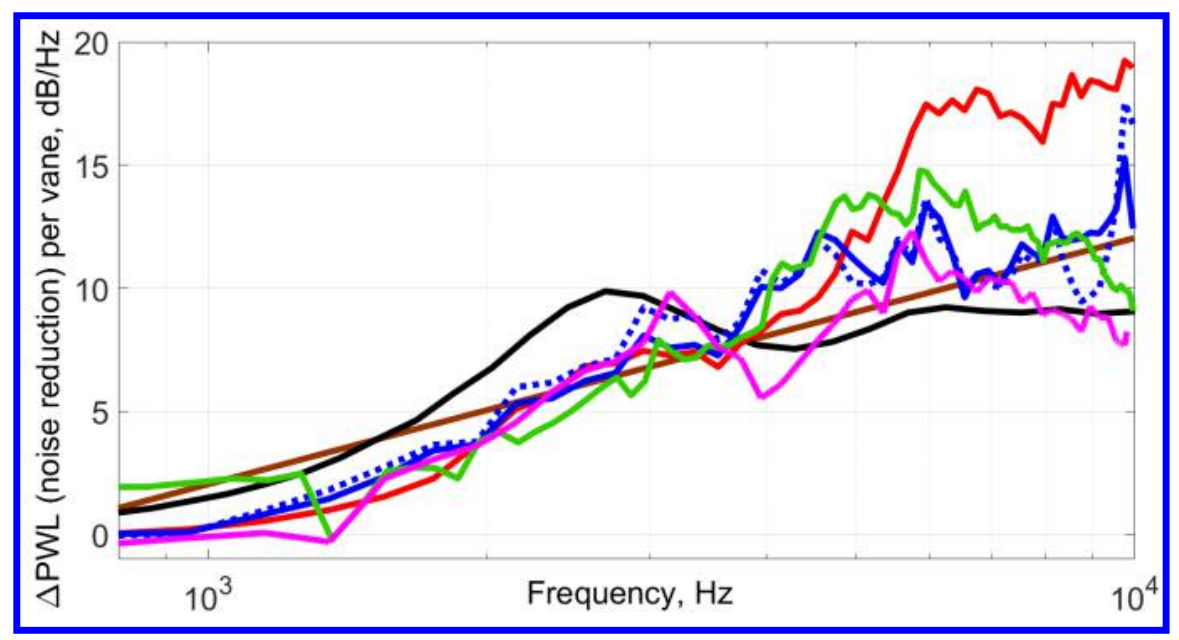

Fig. $23 \quad \triangle$ PWL spectra (downstream radiation). Wiener-Hopf analytical solution in black solid line - and semi-empirical log-law in brown solid line — Numerical solutions for different synthetic turbulence injected in 3-channels computations: - $\left(k_{\xi}, k_{z}=0\right)$, - $\left(k_{\xi}, k_{z}\right),-\left(k_{\xi}, k_{\eta}, k_{z}=0\right),-\left(k_{\xi}, k_{\eta}, k_{z}\right)$.... Numerical solution for a $\left(k_{\xi}, k_{z}\right)$ synthetic turbulence injected in 1-channel computation. A 1 kHz moving average has been applied to raw CAA spectra.

The expected acoustic performances from numerical/analytical/semi-empirical approaches are gathered in Table 3 . There is a very good agreement between the trends drawn from Fig. 23 and the average noise reduction provided in Table 3 However, drawing conclusions from the overall sound power level noise reductions ( $\triangle$ OAPWL) is a bit tricky, since it is influenced by the shape of the baseline noise spectra. Indeed, due to resonance phenomenon associated with periodic boundary condition (more discussed in [5]), some bumps appear in the spectra which tend to over-predict the noise emission around 500 to $1000 \mathrm{~Hz}$, for the 3-channel set-up. The later bump is a bit less visible on a multi-channel computation with a turbulence invariant along the cascade directions, as shown in [5] by Fig. 15. Consequently, in order to give a better idea of the overall noise reduction for the multi-channel computations, the frequency range $1300 \mathrm{~Hz}$ to $9800 \mathrm{~Hz}$ is also considered. In any case, for all the methods a significant $\triangle$ OAPWL (noise reduction) around $6 \mathrm{~dB}$ is expected.

\begin{tabular}{|l|l|l|l|}
\hline & Average $\Delta$ PWL, 0.2 to $9.8 \mathrm{kHz}$ & $\Delta$ OAPWL, 0.2 to $9.8 \mathrm{kHz}$ & $\Delta$ OAPWL, 1.3 to $9.8 \mathrm{kHz}$ \\
\hline WH & 7.3 & 5.5 & 6.8 \\
\hline $10 \log (S t)+10$ & 7.5 & 4.9 & 6.1 \\
\hline CAA $\left(k_{x}, k_{z}=0\right)$ & 11.0 & 5.2 & 6.2 \\
\hline 1 -vane CAA $\left(k_{x}, k_{z}\right)$ & 8.7 & 6.7 & 7.1 \\
\hline CAA $\left(k_{x}, k_{z}\right)$ & 8.6 & 5.4 & 6.4 \\
\hline CAA $\left(k_{x}, k_{y}, k_{z}=0\right)$ & 9.0 & 3.9 & 5.3 \\
\hline CAA $\left(k_{x}, k_{y}, k_{z}\right)$ & 7.2 & 3.5 & 5.7 \\
\hline
\end{tabular}

Table 3 Average $\triangle P W L$ and $\triangle O A P W L$, dB.

Additionally, a parametric study has been performed using the analytical solution (based on the WH technique) in order to evaluate the acoustic performances at the three certification points (approach APP, cutback CUTB and sideline SDL). The obtained results are plotted in Fig. 24. The prediction at $\mathrm{M}=0.3$ (condition used for the CAA) is also given. 
These results show that the noise reduction is shifted towards high frequencies. As already pointed out in [2], the peak-frequency of the PWL reduction roughly follows the one related to the noise emission spectrum, at respective regimes. By the way, the low-noise design remains efficient at low-speed and high-speed flows, and thus well adapted for acoustic certification.

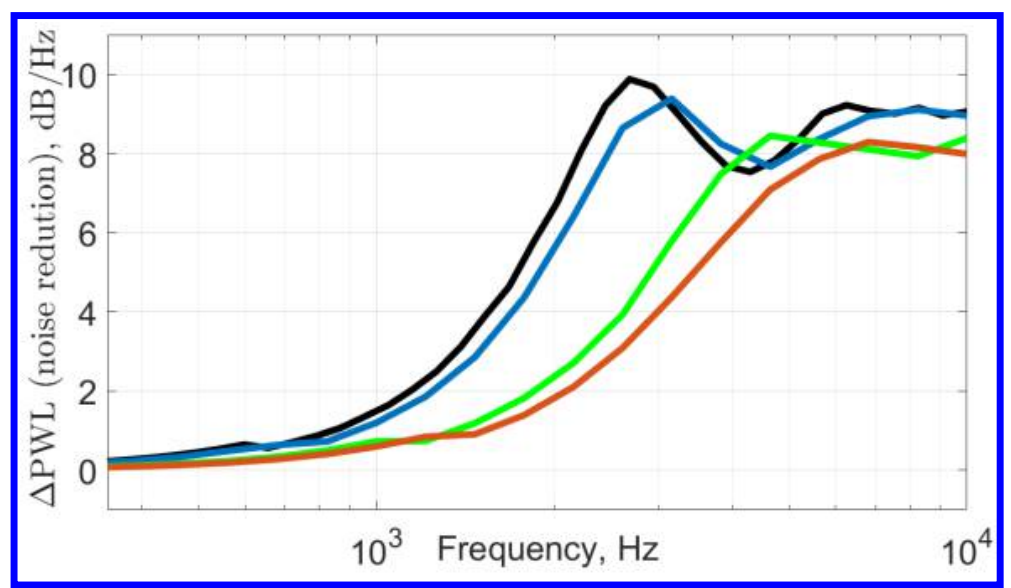

Fig. $24 \quad \triangle P W L$ spectra (downstream radiation) obtained from WH solutions at 3 certification points: APP $(\mathbf{M}=0.34),-$ CUTB $(\mathbf{M}=0.53)$, and - SDL $(\mathbf{M}=0.64)$. WH solution for $\mathbf{M}=0.3$ in black solid line -

\section{Aerodynamic assessment of the serrated design}

\section{A. RANS simulations set-up and aerodynamic performance analyzes}

In addition to aeroacoustic computations, an aerodynamic assessment has been conducted through RANS calculations (performed by R. Barrier) using ONERA code elsA and applying the Jameson spatial scheme and k-1 Smith turbulence model. The full span extension has been considered, with the use of adiabatic walls condition on the vane and end-walls and periodic boundary condition at vane channel sides. In the spanwise direction, 421 points are used, ensuring 30 points per serration wavelength. A view of the CFD grid containing about 5 million cells is shown in Fig. 25a. For the serrated case, the vane skin mesh (see Fig. 25b) is trimmed using the in-house modeler ersatZ. Two operating point conditions have been investigated, at approach (APP) and at the aerodynamic design point (ADP), with inlet Mach number respectively equal to 0.34 and 0.67 , and inlet total pressure and temperature respectively equal to $101325 \mathrm{~Pa}$ and $288.15 \mathrm{~K}$. The inflow turbulence intensity is set equal to $4.5 \%$ and a turbulence viscosity ratio of 0.01 is considered. At APP (selected condition for which the CAA simulations were performed), aerodynamic penalties are found to be acceptable with the first serrated design (mainly achieved for acoustics purpose) as illustrated by Fig. 26 (contour maps of Mach number) and further below in Fig. 29a, related to pressure loss coefficient. In Fig. 26, comparisons of the cut views (taken at the roots of the serration), do not reveal significant changes with only a slight flow separation at the trailing edge suction side. This gives rise to a $0.14 \mathrm{pt}$. deviation for total pressure loss coefficient and $+1.8 \mathrm{deg}$. deviation for outlet flow angle (see summarized results gathered in Table 4 ). 

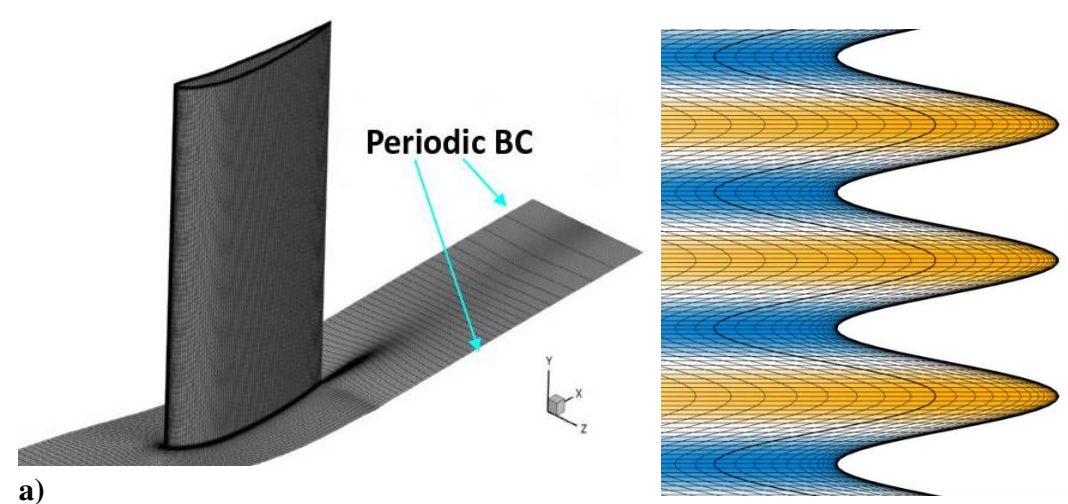

b)

Fig. 25 1-channel mesh of the baseline CFD geometry (on the left) and zoom view of the serrated mesh (on the right).

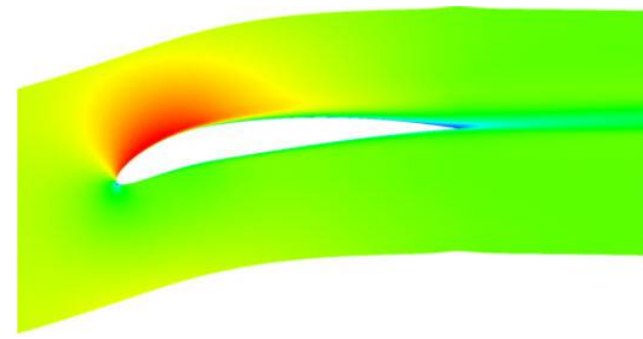

a)

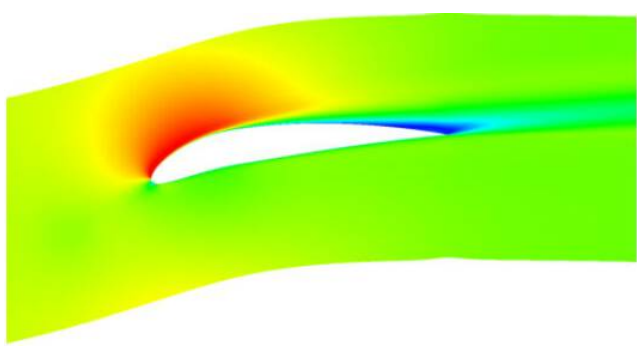

b)

Fig. 26 Mach number (levels between 0.05 and 0.45) at APP. Baseline geometry (on the left) and serrated design with cut at the root (on the right).

However, at the ADP, the aerodynamic performances are significantly deteriorated compared to the reference geometry. Indeed, contrary to the previous observations from Fig. 26, a strong flow separation is clearly shown in Fig. 28b by comparison to the baseline solution in Fig. 28a. This has resulted in the proposal of a second design presented below, consisting in a basic modification of the initial serrated design for aerodynamic purpose only.

\section{B. Proposal of an alternative design to improve aerodynamic behavior at ADP (and APP) operating points}

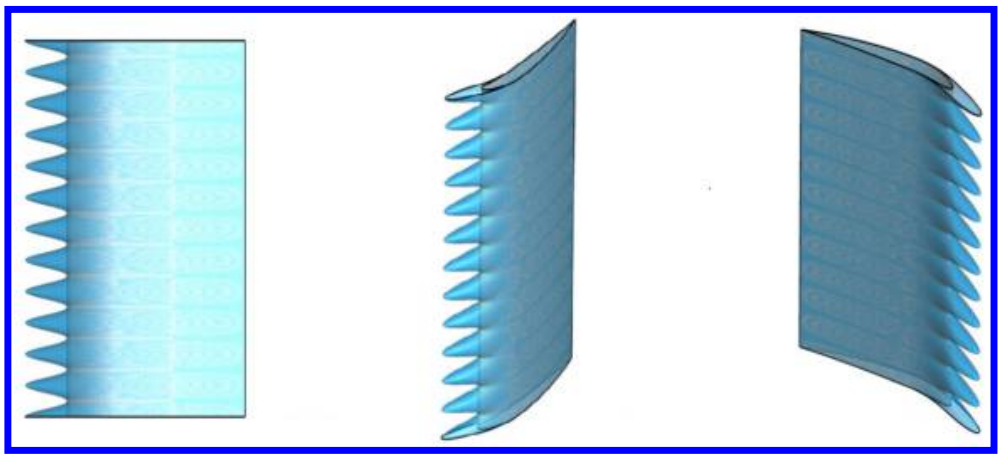

Fig. 27 Views of the baseline geometry (in grey) and the second serrated design (in blue).

In order to limit the aerodynamic penalties, more particularly pointed out at ADP, a second serrated design with an increased averaged chord (the reference chord being set at the roots) has been proposed and simulated too. 3D views of 
this second serrated geometry are depicted in Fig. 27 As the serration parameters $\left(h_{s}, \lambda_{s}\right)$ are unchanged, this design 2 should have almost no impact on acoustic performances assessed with the design 1 . The following results address the aerodynamic performances obtained for both designs in comparison to the reference case. At APP, slightly better performances are obtained with the second design as illustrated by the orange colored pressure loss distribution in Fig. 29a The updated penalties for total pressure loss coefficient and outlet flow angle are found to be respectively reduced to -0.10 pt. and +0.5 deg. with design 2 , see Table 4 At ADP, the strong flow separation observed in Fig. $28 \mathrm{~b}$ is greatly reduced in the presence of design 2 . The overall performances addressed in Table 4 indicate a non-suitable penalty of $-1.6 \mathrm{pt}$. for total pressure loss coefficient and a flow angle deviation of $+3.8 \mathrm{deg}$. with design 1 . These critical values are respectively reduced to $-0.6 \mathrm{pt}$. and $+0.8 \mathrm{deg}$. with design 2 , which is a quite valuable improvement. These positive effects are clearly pointed out on the spanwise profiles of pressure loss coefficient obtained for both designs, compared to the reference solution in Fig. 29b at ADP. The benefit of using an increased mean chord is clearly highlighted, the design 2 being able to reduce the penalties all over the span, with much more acceptable deviations.

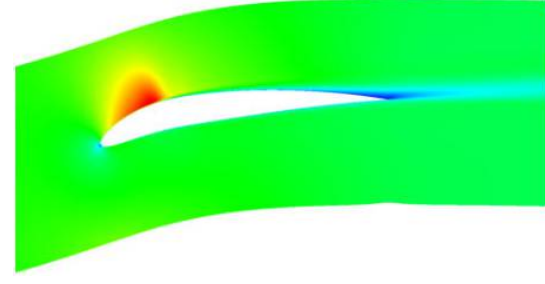

a)

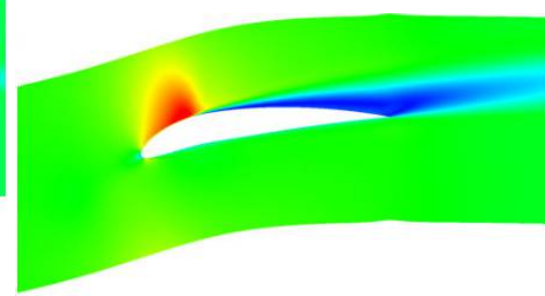

b)

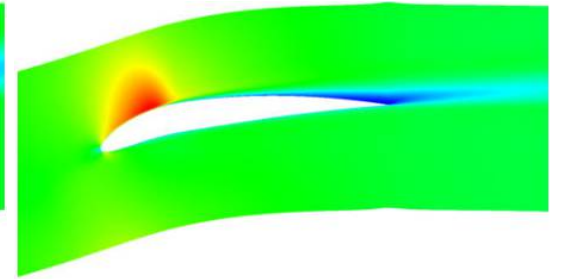

c)

Fig. 28 Mach number (levels between 0.1 and 1.2) contour maps cuts at ADP. Baseline geometry (on the left), serrated design 1 (in the middle), and serrated design 2 (in the right) with cuts at the roots.

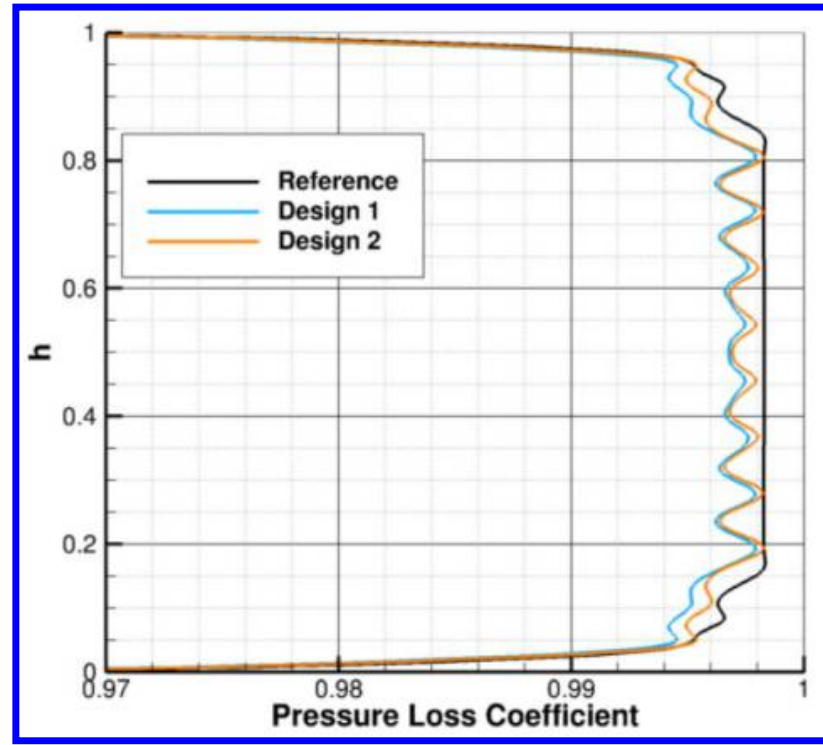

a)

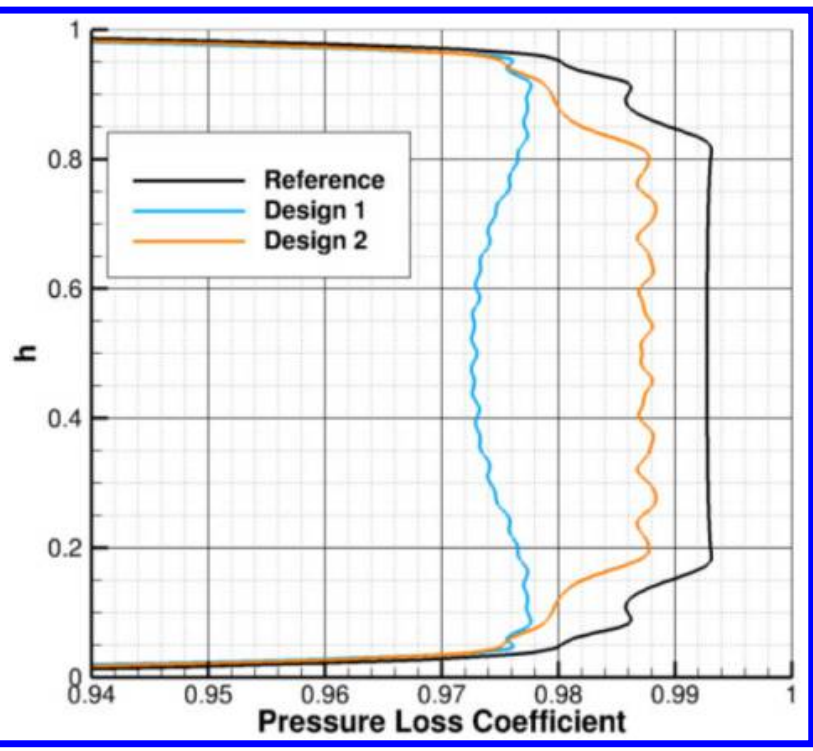

b)

Fig. 29 Pressure loss distributions along the dimensionless spanwise coordinate $h=z / L_{z}$. At APP (on the left) and ADP (on the right). 


\begin{tabular}{|l|l|l|l|}
\hline & baseline (reference) & design 1 & design 2 \\
\hline Total pressure loss coefficient at APP & 0.9970 & $0.9956(-0.14 \mathrm{pt})$. & $0.9960(-0.10 \mathrm{pt})$. \\
Total pressure loss coefficient at ADP & 0.9880 & $0.9720(-1.60 \mathrm{pt})$. & $0.9820(-0.60 \mathrm{pt})$. \\
\hline Outlet angle (remaining swirl) at APP & $2.1^{\circ}$ & $3.9^{\circ}\left(+1.8^{\circ}\right)$ & $2.6^{\circ}\left(+0.5^{\circ}\right)$ \\
Outlet angle (remaining swirl) at ADP & $2.5^{\circ}$ & $6.3^{\circ}\left(+3.8^{\circ}\right)$ & $3.3^{\circ}\left(+0.8^{\circ}\right)$ \\
\hline
\end{tabular}

Table 4 Summary of the aerodynamic performances of baseline, serrated design 1, and design 2 . Difference to the reference value in parenthesis.

\section{Conclusion}

The ability to implement a fully three-dimensional turbulence with a limited computational cost has been demonstrated in this study. Wall and periodic boundary conditions have raised important challenges. To address the latter issues, a variant of the usual generation process based on the energy spectrum, similarly to [15], has been proposed. Adequately defined weighting functions have been introduced to obtain a divergence free turbulence in the presence of wall BC. From a methodological point of view, this work has provided some additional insight on the set-up of CAA computations, following on preliminary CAA simulations performed at ONERA on the InnoSTAT configuration [5]. One may conclude that multi-channel computations with at least a $\left(k_{\xi}, k_{\eta}, k_{z}=0\right)$ turbulence are needed to reproduce the most accurately the noise spectra on multi-channel configurations in medium and high frequencies. The convergence in low frequencies is ensured only by taking into account a large amount of airfoils as shown in [5, 31]. If one is only interested in the relative noise reduction, a one channel simulation with a $\left(k_{\xi}, k_{z}\right)$ turbulence structure appears appropriate. However, in order to take into account very properly both cascade and serration effects, a 3D synthetic turbulence might represent the best choice. The only drawback of a multi-channel computation with a 3D turbulence is that an important number of vanes has to be taken into account in order to damp the bump in low frequency of the acoustic spectra associated with periodic boundary conditions. Thus, an alternative is to consider multi-channel computations with $\left(k_{\xi}, k_{z}\right)$ turbulence as usually performed at ONERA [1, 2], even if the spectra are a bit altered in medium and high frequencies. In any case, the new fully 3D turbulence methodology might be of particular interest for cases where both $k_{\eta}$ and $k_{z}$ are likely to strongly influence the acoustic sources. In parallel, a low-noise design with leading edge serrations has been proposed for which around 4 to $6 \mathrm{~dB}$ overall noise reduction can be expected at the 3 certification points, with a good agreement between the noise reduction spectra from the different methods. Finally, aerodynamic performances of the serrated design were evaluated. At the approach point, the design offers acceptable performance losses. However, at the ADP operating point, the aerodynamic penalties raise the need for an improved variant of the initial design. This second design, involving a larger mean chord, should not modify the acoustic prediction since the leading edge sinusoidal shape has been preserved. Even better, the improved aerodynamics might reduce the additional self-noise sources compared to the first design.

As mentioned in the introduction, the LBM is an affordable high-fidelity approach that might be complementary to the present hybrid CFD/CAA methods. In order to compare both, LBM calculations using the ProLB code are underway at ONERA on these baseline and low-noise cascade geometries, including the full ECL cascade rig set-up [4].

\section{Appendix}

\section{A. An algorithm to determine the direction of the velocity for 3D turbulence structures}

Let us consider the wavenumber $\boldsymbol{k}=\left(k_{\xi, l}, k_{\eta, l m}, k_{z, n}\right)^{T}$ in the coordinate system $(\xi, \eta, z)$. The goal is to build on orthonormal basis $\left(\boldsymbol{k}_{\boldsymbol{a}}, \boldsymbol{k}_{\boldsymbol{b}}, \boldsymbol{k}_{\boldsymbol{c}}\right)$ from $\boldsymbol{k}$ in order to determine $\boldsymbol{\sigma}_{\boldsymbol{l m} \boldsymbol{n}}$ such as $\boldsymbol{k} \cdot \boldsymbol{\sigma}_{\boldsymbol{l m} \boldsymbol{n}}=0$. The main steps of the procedures are mentioned below with $\alpha_{l m n}$ a random phase term sorted for each mode $(l, m, n)$.

1) $k_{c}=\frac{k}{\|k\|}$ 
2) Supposing that $k_{\xi}>0\left\{\begin{array}{l}\boldsymbol{k}_{\boldsymbol{a}}=\left(-\frac{k_{\eta}^{2}+k_{z}^{2}}{k_{\xi}}, k_{\eta}, k_{z}\right)_{(\xi, \eta, z)}^{T}, \text { if }\left|k_{\eta}\right|+\left|k_{z}\right| \neq 0 \\ \boldsymbol{k}_{\boldsymbol{a}}=\left(0, k_{\eta}, 0\right)_{(\xi, \eta, z)}^{T}, \text { if }\left|k_{\eta}\right|+\left|k_{z}\right|=0\end{array}\right.$

3) $\boldsymbol{k}_{\boldsymbol{a}}=\frac{\boldsymbol{k}_{\boldsymbol{a}}}{\left\|\boldsymbol{k}_{\boldsymbol{a}}\right\|}$ and $\boldsymbol{k}_{\boldsymbol{b}}=\frac{\boldsymbol{k}_{\boldsymbol{b}}}{\left\|\boldsymbol{k}_{\boldsymbol{b}}\right\|}$

4) $\boldsymbol{k}_{\boldsymbol{b}}=\boldsymbol{k}_{\boldsymbol{c}} \wedge \boldsymbol{k}_{\boldsymbol{a}}$

5) $\boldsymbol{\sigma}_{\text {lmn }}=\cos \left(\alpha_{l m n}\right) \boldsymbol{k}_{\boldsymbol{a}}+\sin \left(\alpha_{l m n}\right) \boldsymbol{k}_{\boldsymbol{b}}$, with $\alpha_{l m n} \in[0,2 \pi]$

B. Link between formulations based on the turbulence energy spectrum and the velocity autocorrelation spectra

For the sake of simplicity, let us assume that $\beta_{c}=0$. In order to achieve the comparison between the formulations, an amplitude of a given mode $u_{i}^{\prime}=2 \sqrt{E(k) \Delta k} \sigma_{i}=2 \sqrt{E(k) /\left(2 \pi k^{2}\right) \Delta k_{x} \Delta k_{y} \Delta k_{z}} \sigma_{i}$ is considered. The directivity of the velocity is given by the vector $\sigma$ which satisfies Eq. 24, , following Appendix. A.

$$
\boldsymbol{\sigma}=\cos (\alpha) \frac{k}{k^{2} \sqrt{k_{y}^{2}+k_{z}^{2}}}\left(\begin{array}{c}
-\left(k_{y}^{2}+k_{z}^{2}\right) \\
k_{x} k_{y} \\
k_{x} k_{z}
\end{array}\right)+\sin (\alpha) \frac{1}{k^{2} \sqrt{k_{y}^{2}+k_{z}^{2}}}\left(\begin{array}{c}
0 \\
-k_{z} k^{2} \\
k_{y} k^{2}
\end{array}\right)
$$

The next step is to consider the mean value (denoted by the subscript mean) of the squared norm of the vector $\sigma$ from a statistical point of view, considering an infinite number of $\alpha$ randomly sorted.

$$
\begin{array}{ll}
\sigma_{1}^{2}=\cos ^{2}(\alpha) \frac{1}{k^{2}}\left(k_{y}^{2}+k_{z}^{2}\right) & \Rightarrow \sigma_{1, \text { mean }}^{2}=\frac{1}{2} \frac{1}{k^{2}}\left(k_{y}^{2}+k_{z}^{2}\right)=\frac{1}{2}\left(1-\frac{k_{x}^{2}}{k^{2}}\right) \\
\sigma_{2}^{2}=\frac{1}{k^{4}\left(k_{y}^{2}+k_{z}^{2}\right)}\left(\cos (\alpha) k_{x} k_{y} k-\sin (\alpha) k_{z} k^{2}\right)^{2} \Rightarrow \sigma_{2, \text { mean }}^{2}=\frac{1}{2} \frac{1}{k^{2}}\left(k_{x}^{2}+k_{z}^{2}\right)=\frac{1}{2}\left(1-\frac{k_{y}^{2}}{k^{2}}\right) \\
\sigma_{3}^{2}=\frac{1}{k^{4}\left(k_{y}^{2}+k_{z}^{2}\right)}\left(\cos (\alpha) k_{x} k_{z} k+\sin (\alpha) k_{y} k^{2}\right)^{2} \Rightarrow \sigma_{3, \text { mean }}^{2}=\frac{1}{2} \frac{1}{k^{2}}\left(k_{x}^{2}+k_{y}^{2}\right)=\frac{1}{2}\left(1-\frac{k_{z}^{2}}{k^{2}}\right)
\end{array}
$$

The expression of averaged (mean) velocities, with respect to $\alpha$, can now be expanded.

$$
\begin{aligned}
& u_{1, \text { mean }}^{\prime 2}=4 \frac{E(k)}{4 \pi k^{2}}\left(1-\frac{k_{x}^{2}}{k^{2}}\right) \Delta k_{x} \Delta k_{y} \Delta k_{z} \\
& u_{2, \text { mean }}^{\prime 2}=4 \frac{E(k)}{4 \pi k^{2}}\left(1-\frac{k_{y}^{2}}{k^{2}}\right) \Delta k_{x} \Delta k_{y} \Delta k_{z} \\
& u_{3, \text { mean }}^{\prime 2}=4 \frac{E(k)}{4 \pi k^{2}}\left(1-\frac{k_{z}^{2}}{k^{2}}\right) \Delta k_{x} \Delta k_{y} \Delta k_{z}
\end{aligned}
$$

If one considers the squared amplitude of the average statistical velocities $\left(u_{i, \text { mean }}^{\prime}=2 \sqrt{\varphi_{i i} \Delta k_{x} \Delta k_{y} \Delta k_{z}}\right)$, the formulations which would have been obtained using autocorrelation spectra $\varphi_{i i}=E(k) /\left(4 \pi k^{2}\right)\left(1-k_{i}^{2} / k^{2}\right)$ are recovered. To conclude, it has been demonstrated that if an infinite number of modes is sorted, the two formulations (relying on the energy spectrum or the autocorrelation velocity spectra) tend towards the same limit.

\section{Determining conditions under which a divergence free formulation can be achieved}

The choice has been made to alter the $u_{z}^{\prime}$ component, with the aim of achieving a divergence free formulation.

$$
\frac{\partial u_{z}^{\prime}}{\partial z}=-f_{w}(z) A \sin (\boldsymbol{k} \cdot \boldsymbol{X}-\omega t+\psi) k_{z} \sigma_{z}+f_{w}^{\prime}(z) A \cos (\boldsymbol{k} \cdot \boldsymbol{X}-\omega t+\psi) \sigma_{z}-g_{w}^{\prime}(z) A \sigma_{z}
$$


From Eqs. 21] and 25], one can obtain that $g_{w}^{\prime}(z)=f_{w}^{\prime}(z) \cos (\boldsymbol{k} \cdot \boldsymbol{X}-\omega t+\psi)$. More precisely, $g_{w}(z)$ is a piecewise function likewise $f_{w}(z)$, defined as follows,

$$
\begin{cases}\forall z \in\left[z_{\text {min }}, z_{\text {min }}+L_{w}\right], & g_{w}^{\prime}(z)=\frac{\pi}{2 L_{w}} \sin \left(\pi \frac{z-z_{\min }}{L_{w}}\right) \cos (\boldsymbol{k} \cdot \boldsymbol{X}-\omega t+\psi) \\ \forall z \in\left[z_{\text {min }}+L_{w}, z_{\text {max }}-L_{w}\right], & g_{w}^{\prime}(z)=0 \\ \forall z \in\left[z_{\text {max }}-L_{w}, z_{\text {max }}\right], & g_{w}^{\prime}(z)=\frac{\pi}{2 L_{w}} \sin \left(\pi \frac{z-z_{\text {max }}}{L_{w}}\right) \cos (\boldsymbol{k} \cdot \boldsymbol{X}-\omega t+\psi)\end{cases}
$$

The idea of the following paragraphs is to demonstrate the conditions under which the previous system of equations (Eq. (26) can be satisfied taking into account that both functions $f_{w}$ and $g_{w}$ are $C^{1}$ and cancel in $z_{\min }$ and $z_{\max }$. Let us consider that $k_{z} \neq \pm \pi / L_{w}$ (the case $k_{z}= \pm \pi / L_{w}$ which is not detailed here and leads to the equality $\sin \left(k_{x} x+k_{y} y+\pi / L_{w} z_{\min }-\omega t+\psi\right)=0$, which can not be ensured for every set of variables).

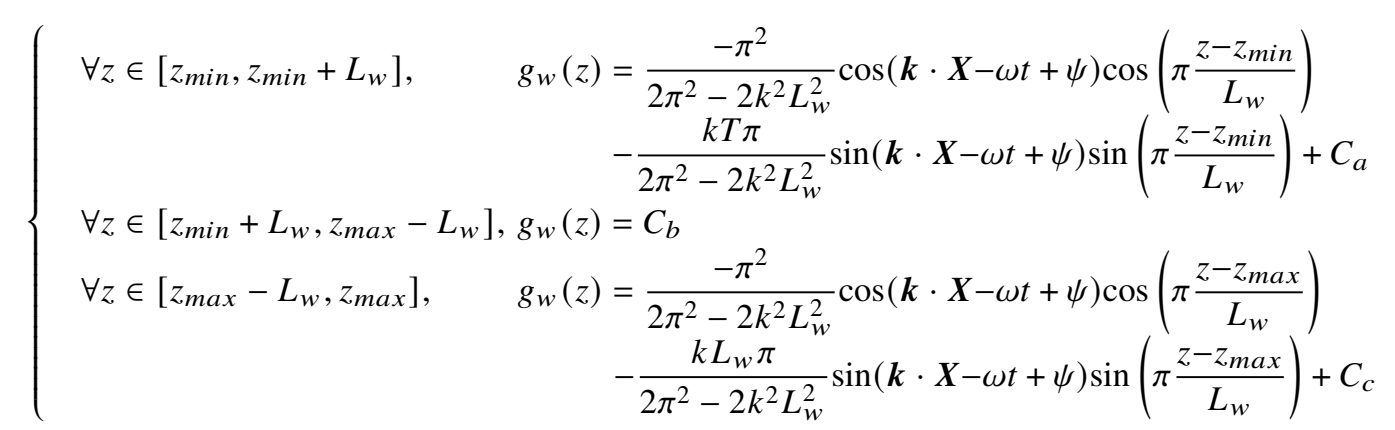

The integrated form of Eq. (26) is given by Eq. (27), where three constants $C_{a}, C_{b}$, and $C_{c}$ need to be determined. The functions $f_{w}$ and $g_{w}$ have to be damped near the boundaries, for example at $z_{\min }, g_{w}\left(z_{\min }\right)=0$. Thus, the constant $C_{a}$ satisfies,

$$
C_{a}=\frac{\pi^{2}}{2 \pi^{2}-2 k^{2} L_{w}^{2}} \cos \left(k_{x} x+k_{y} y+k_{z} z_{\min }-\omega t+\psi\right)
$$

The continuity has to be ensured in $z=z_{\min }+L_{w}$, leading to $C_{b}=g_{w}\left(z_{\min }+L_{w}\right)$.

$$
C_{b}=\frac{\pi^{2}}{2 \pi^{2}-2 k^{2} L_{w}^{2}} \cos \left(k_{x} x+k_{y} y+k_{z}\left(z_{\text {min }}+L_{w}\right)-\omega t+\psi\right)+\frac{\pi^{2}}{2 \pi^{2}-2 k^{2} L_{w}^{2}} \cos \left(k_{x} x+k_{y} y+k_{z} z_{\text {min }}-\omega t+\psi\right)
$$

Thus, the constant $C_{b}$ can be expressed by Eq. 29].

$$
C_{c}=\frac{\pi^{2}}{2 \pi^{2}-2 k^{2} L_{w}^{2}} \cos \left(k_{x} x+k_{y} y+k_{z} z_{\max }-\omega t+\psi\right)
$$

Likewise at $z=z_{\max }, g_{w}\left(z_{\max }\right)=0$ which leads to Eq. 30.

$$
C_{b}=\frac{\pi^{2}}{2 \pi^{2}-2 k^{2} L_{w}^{2}} \cos \left(k_{x} x+k_{y} y+k_{z}\left(z_{\max }-L_{w}\right)-\omega t+\psi\right)+\frac{\pi^{2}}{2 \pi^{2}-2 k^{2} L_{w}^{2}} \cos \left(k_{x} x+k_{y} y+k_{z} z_{\max }-\omega t+\psi\right)
$$

Thereafter, $C_{b}=g_{w}\left(z_{\max }-L_{w}\right)$ and $C_{b}$ has also to satisfy Eq. [31).

$\cos \left(k_{x} x+k_{y} y+k_{z} z_{\text {min }}+\frac{k_{z} L_{w}}{2}-\omega t+\psi\right) \cos \left(\frac{k_{z} L_{w}}{2}\right)=\cos \left(k_{x} x+k_{y} y+k_{z} z_{\min }+k_{z} L_{z}-\frac{k_{z} L_{w}}{2}-\omega t+\psi\right) \cos \left(\frac{k_{z} L_{w}}{2}\right)$

The constraint that $C_{b}=g_{w}\left(z_{\min }+L_{w}\right)=g_{w}\left(z_{\max }-L_{w}\right)$, imposes that Eq. 32) has to be verified. 


\section{Acknowledgments}

The present work was conducted through a collaboration within the CleanSky2 project InnoSTAT (865007), and has received fundings from European Union's Horizon 2020 research and innovation program in the frame of ADEC project of CS2 LPA-IADP.

\section{References}

-[1] Cader, A., Polacsek, C., Garrec, T. L., Barrier, R., Benjamin, F., and Jacob., M., "Numerical prediction of rotor-stator interaction noise using 3D CAA with synthetic turbulence injection," Proc. of the AIAA/CEAS Aeroacoustics Conference, Atlanta, United States, 2018.

[2] Polacsek, C., Cader, A., Buszyk, M., Barrier, R., Gea-Aguilera, F., and Posson, H., "Aeroacoustic Design and Broadband Noise Predictions of a Fan Stage with Serrated Outlet Guide Vanes," Physics of fluids, Vol. 32, No. 10, 2020, p. 107107.

[3] Casalino, D., Avallone, F., Gonzalez-Martino, I., and Ragni, D., "Aeroacoustic study of a wavy stator leading edge in a realistic fan/OGV stage," Journal of Sound and Vibration, Vol. 442, 2018. https://doi.org/10.1016/j.jsv.2018.10.057.

[4] Buszyk, M., Polacsek, C., Garrec, T. L., and Barrier, R., "Lattice Boltzmann simulations in a rectilinear cascade configuration for the turbulence-airfoil interaction noise evaluation and reduction through serrated leading edges," to be presented at Euronoise 2021 Conference (submitted and accepted abstract), 2021.

[5] Buszyk, M., Polacsek, C., and Garrec, T. L., "Assessment of a CAA methodology for turbulence-cascade interaction noise prediction and reduction from serrated airfoils," eForum Acusticum 2020, Lyon, France, 2020, pp. $2961-2968$. https://doi.org/10.48465/fa.2020.1141. URL https://hal.archives-ouvertes.fr/hal-03229464

- [6] Kim, J. W., and Haeri, S., "An advanced synthetic eddy method for the computation of aerofoil-turbulence interaction noise," Journal of Computational Physics, Vol. 287, 2015, pp. 1-17. https://doi.org/https://doi.org/10.1016/j.jcp.2015.01.039. URL https://www.sciencedirect.com/science/article/pii/S0021999115000534

[7] Gea-Aguilera, F., Gill, J., and Zhang, X., "Synthetic Turbulence Methods for Computational Aeroacoustic Simulations of Leading Edge Noise," Computers \& Fluids, Vol. 157, 2017, pp. 240-252. https://doi.org/10.1016/j.compfluid.2017.08.039.

-[8] Gea-Aguilera, F., Gill, J., Angland, D., and Zhang, X., "Wavy Leading Edge Airfoils Interacting with Anisotropic Turbulence," 23rd AIAA/CEAS Aeroacoustics Conference, 2017. https://doi.org/10.2514/6.2017-3370, URL https://arc.aiaa.org/doi/abs/10. 2514/6.2017-3370

- [9] Gea-Aguilera, F., Karve, R., Gill, J., Zhang, X., and Angland, D., "On the effects of anisotropic turbulence on leading edge noise," Journal of Sound and Vibration, Vol. 495, 2021, p. 115895. https://doi.org/https://doi.org/10.1016/j.jsv.2020.115895. URL https://www.sciencedirect.com/science/article/pii/S0022460X20307343.

-[10] Ewert, R., Dierke, J., Siebert, J., Neifeld, A., Appel, C., Siefert, M., and Kornow, O., "CAA broadband noise prediction for aeroacoustic design," Journal of Sound and Vibration, 2011.

-[11] Kissner, C. A., and Guérin, S., Fan Broadband Noise Prediction for the ACAT1 Fan Using a Three-Dimensional Random Particle Mesh Method, 2020. https://doi.org/10.2514/6.2020-2520. URL/https://arc.aiaa.org/doi/abs/10.2514/6.2020-2520

-[12] Kissner, C. A., and Guérin, S., "Comparison of Predicted Fan Broadband Noise Using a Two- versus a Three-Dimensional Synthetic Turbulence Method," Journal of Sound and Vibration, 2021, p. 116221. https://doi.org/10.1016/j.jsv.2021.116221

-[13] Kraichnan, R., "Diffusion by a random velocity field," Physics of Fluids, Vol. 13, 1970, pp. 22-31.

-[14] Bechara, W., Bailly, C., Lafon, P., and Candel, S., "Stochastic approach to noise modeling for free turbulent flows," AIAA Journal, Vol. 32, 1994, pp. 455-463.

[15] Gill, J. S., Zhang, X., and Joseph, P. F., "Single velocity-component modeling of leading edge turbulence interaction noise," The Journal of the Acoustical Society of America, Vol. 137, No. 6, 2015, pp. 3209-3220. https://doi.org/10.1121/1.4921547. URL https://doi.org/10.1121/1.4921547

-[16] Amiet, R. K., "High frequency thin-airfoil theory for subsonic flow," AIAA Journal, Vol. 14, No. 8, 1976, pp. $1076-1082$. https://doi.org/10.2514/3.7187.

- [17] Niedoba, P., Jicha, M., and Čermák, L., "On stochastic inlet boundary condition for unsteady simulations," EPJ Web of Conferences, Vol. 67, 2014. https://doi.org/10.1051/epjconf/20146702082. 
[18] Reboul, G., Cader, A., Polacsek, C., Garrec, T. L., Barrier, R., and Nasr, N. B., CAA Prediction of Rotor-Stator Interaction Using Synthetic Turbulence: Application to a Low-Noise Serrated OGV, 2017. https://doi.org/10.2514/6.2017-3714 URL https://arc.aiaa.org/doi/abs/10.2514/6.2017-3714

[19] Paruchuri, C., Joseph, P., Narayanan, S., Vanderwel, C., Turner, J., Kim, J. W., and Ganapathisubramani, B., "Performance and mechanism of sinusoidal leading edge serrations for the reduction of turbulence-aerofoil interaction noise," Journal of Fluid Mechanics, Vol. 818, 2017, p. 435-464. https://doi.org/10.1017/jfm.2017.141.

[20] Ayton, L., and Paruchuri, C., "An analytical and experimental investigation of aerofoil-turbulence interaction noise for plates with spanwise-varying leading edges," Journal of Fluid Mechanics, Vol. 865, 2019, pp. 137-168.

[21] Finez, A., "Etude expérimentale du bruit de bord de fuite à large bande d'une grille d'aubes linéaire et de sa réduction par dispositifs passifs," Ph.D. thesis, Ecole centrale de Lyon, France, 2012.

[22] Redonnet, S., Manoha, E., and Sagaut, P., Numerical simulation of propagation of small perturbations interacting with flows and solid bodies, 2001. https://doi.org/10.2514/6.2001-2223. URL https://arc.aiaa.org/doi/abs/10.2514/6.2001-2223

[23] Clair, V., “Calcul numérique de la réponse acoustique d'un aubage soumis à un sillage turbulent,” Ph.D. thesis, Ecole centrale de Lyon, France, 2013.

-[24] Tam, C. K. W., and Dong, Z., "Radiation and outflow boundary conditions for direct computation of acoustic and flow disturbances in a nonuniform mean flow," Journal of Computational Acoustics, Vol. 04, No. 02, 1996, pp. $175-201$. https://doi.org/10.1142/S0218396X96000040. URL https://doi.org/10.1142/S0218396X96000040.

[25] Blazek, J., Computational Fluid Dynamics 3rd Edition. Principles and Applications., Butterworth-Heinemann, Germany, 2015.

[26] Liepmann, H. W., Laufer, J., and Liepmann, K., “On the Spectrum of Isotropic Turbulence,” National Advisory Committee for Aeronautics, 1951.

- [27] Clair, V., Polacsek, C., Garrec, T. L., Reboul, G., Gruber, M., and Joseph, P., "Experimental and Numerical Investigation of Turbulence-Airfoil Noise Reduction Using Wavy Edges,” AIAA Journal, Vol. 51, 2013, pp. 2695-2713. https://doi.org/10.2514/ $1 . J 052394$

[28] Glegg, S., "The response of a swept blade row to a three-dimensional gust," Journal of Sound and Vibration, Vol. 227, 1999, pp. 29-64.

[29] Hanson, D., and Horan, K., "Turbulence/cascade interaction - Spectra of inflow, cascade response, and noise," 4th AIAA/CEAS Aeroacoustics Conference, 1998.

[30] Blandeau, V., Joseph, P., Jenkins, G., and Powles, C., "Comparison of sound power radiation from isolated airfoils and cascades in a turbulent flow," The Journal of the Acoustical Society of America, Vol. 129, 2011, pp. 3521-30.

[31] Gea-Aguilera, F., “Aerodynamic and aeroacoustic modelling of engine fan broadband noise," Ph.D. thesis, University of Southampton, England, 2017.

[32] Paruchuri, C., "Aerofoil geometry effects on turbulence interaction noise," Ph.D. thesis, University of Southampton, April 2017. URL https://eprints.soton.ac.uk/415884/ 